\title{
Existence of travelling wave solutions in delayed reaction-diffusion systems with applications to diffusion-competition systems
}

\author{
Wan-Tong $\mathrm{Li}^{1}$, Guo Lin ${ }^{1}$ and Shigui Ruan ${ }^{2}$ \\ ${ }^{1}$ School of Mathematics and Statistics, Lanzhou University, Lanzhou, Gansu 730000, \\ People's Republic of China \\ 2 Department of Mathematics, University of Miami, PO Box 249085, Coral Gables, \\ FL 33124-4250, USA
}

Received 1 June 2005, in final form 4 April 2006

Published 15 May 2006

Online at stacks.iop.org/Non/19/1253

Recommended by B Eckhardt

\begin{abstract}
This paper is concerned with the existence of travelling wave solutions in a class of delayed reaction-diffusion systems without monotonicity, which concludes two-species diffusion-competition models with delays. Previous methods do not apply in solving these problems because the reaction terms do not satisfy either the so-called quasimonotonicity condition or non-quasimonotonicity condition. By using Schauder's fixed point theorem, a new cross-iteration scheme is given to establish the existence of travelling wave solutions. More precisely, by using such a new cross-iteration, we reduce the existence of travelling wave solutions to the existence of an admissible pair of upper and lower solutions which are easy to construct in practice. To illustrate our main results, we study the existence of travelling wave solutions in two delayed two-species diffusion-competition systems.
\end{abstract}

Mathematics Subject Classification: 35K57, 35R20, 92D25

\section{Introduction}

In recent years, great attention has been paid to the study of the existence of travelling waves in reaction-diffusion systems with delays. In a pioneering work, Schaaf [27] systematically studied two scalar reaction-diffusion equations with a single discrete delay for the so-called Huxley nonlinearity as well as Fisher nonlinearity by using the phase space analysis, the maximum principle for parabolic functional differential equations and the general theory for ordinary functional differential equations. For reaction-diffusion systems with quasimonotonicity (QM) and a single discrete delay, Zou and $\mathrm{Wu}$ [36] established the existence 
of travelling wave fronts by first truncating the unbounded domain and then passing to a limit. $\mathrm{Wu}$ and Zou [33] further considered more general reaction-diffusion systems with a single delay of the form

$$
\frac{\partial}{\partial t} u(x, t)=D \frac{\partial^{2}}{\partial x^{2}} u(x, t)+f\left(u_{t}(x)\right),
$$

where $t \in \mathbb{R}, x \in \mathbb{R}, D=\operatorname{diag}\left(d_{1}, \cdots, d_{n}\right), d_{i}>0, i=1, \cdots n, f \in C\left([-\tau, 0], \mathbb{R}^{n}\right)$ is continuous and satisfies $f(\hat{\mathbf{0}})=f(\hat{\mathbf{K}})=\mathbf{0}$; here $\hat{u}$ denotes the constant vector function on $[-\tau, 0]$ taking the value $u$, and for any fixed $x \in \mathbb{R}, u_{t}(x) \in C\left([-\tau, 0], \mathbb{R}^{n}\right)$ is defined by $u_{t}(x)=u(t+\theta, x), \theta \in[-\tau, 0]$. If the reaction term $f$ satisfies either the QM condition

(QM) there exists a matrix $\beta=\operatorname{diag}\left(\beta_{1}, \beta_{2}, \ldots, \beta_{n}\right)$ with $\beta_{i} \geqslant 0$ such that

$$
f(\Phi)-f(\Psi)+\beta(\Phi(0)-\Psi(0)) \geqslant 0
$$

for $\Phi, \Psi \in C\left([-\tau, 0], \mathbb{R}^{n}\right)$ with $\mathbf{0} \leqslant \Psi(s) \leqslant \Phi(s) \leqslant \mathbf{K}, s \in[-\tau, 0]$

or the non-quasimonotonicity $\left(\mathrm{QM}^{*}\right)$ condition

$\left(\mathrm{QM}^{*}\right)$ there exists a matrix $\beta=\operatorname{diag}\left(\beta_{1}, \beta_{2}, \ldots, \beta_{n}\right)$ with $\beta_{i} \geqslant 0$ such that

$$
f(\Phi)-f(\Psi)+\beta(\Phi(0)-\Psi(0)) \geqslant 0
$$

for $\Phi, \Psi \in C\left([-\tau, 0], \mathbb{R}^{n}\right)$ with (i) $\mathbf{0} \leqslant \Psi(s) \leqslant \Phi(s) \leqslant \mathbf{K}, s \in[-\tau, 0]$ and (ii) $e^{\beta s}(\Phi(s)-$ $\Psi(s))$ is non-decreasing in $s \in[-\tau, 0]$;

then some existence results are established for travelling wave fronts connecting the trivial equilibrium $\mathbf{0}$ and the non-trivial equilibrium $\mathbf{K}$, where the well-known monotone iteration techniques for elliptic systems with advanced arguments are used [20, 23]. The results are applicable not only to delayed scalar equations (Lan and Wu [19]) but also to delayed systems, such as delayed diffusion-cooperation systems (Huang and Zou [14]) and the delayed Belousov-Zhabotinskii model (Huang and Zou [15]). Following Wu and Zou [33], Ma [22] employed the Schauder's fixed point theorem to an operator used in Wu and Zou [33] in a properly chosen subset in the Banach space $C\left(\mathbb{R}, \mathbb{R}^{n}\right)$ equipped with the so-called exponential decay norm. The subset is constructed in terms of a pair of upper-lower solutions, which is less restrictive than the upper-lower solutions required in [33]. This makes the search for the pair of upper-lower solutions slightly easier. Since Ma [22] only considered delayed systems with quasimonotone reaction terms, Huang and Zou [15] extended the results of Ma [22] to a class of delayed systems with $\mathrm{QM}^{*}$ reaction terms. For related results on reaction-diffusion equations with non-local delays, we refer to Ashwin et al [1], Al-Omari and Gourly [2], Billingham [3], Li, Ruan and Wang [21], Wang, Li and Ruan [31] and references cited therein.

However, it is quite common that the reaction term in a model system arising from a practical problem may not satisfy either the QM condition or the QM* condition. Two typical and important examples are the two species competition systems [26,32]:

$$
\left\{\begin{array}{l}
\frac{\partial}{\partial t} u_{1}(x, t)=d_{1} \frac{\partial^{2}}{\partial x^{2}} u_{1}(x, t)+r_{1} u_{1}(x, t)\left[1-a_{1} u_{1}(x, t)-b_{1} u_{2}\left(x, t-\tau_{1}\right)\right], \\
\frac{\partial}{\partial t} u_{2}(x, t)=d_{2} \frac{\partial^{2}}{\partial x^{2}} u_{2}(x, t)+r_{2} u_{2}(x, t)\left[1-b_{2} u_{1}\left(x, t-\tau_{2}\right)-a_{2} u_{2}(x, t)\right]
\end{array}\right.
$$

and

$$
\left\{\begin{array}{l}
\frac{\partial}{\partial t} u_{1}(x, t)=d_{1} \frac{\partial^{2}}{\partial x^{2}} u_{1}(x, t)+r_{1} u_{1}(x, t)\left[1-a_{1} u_{1}\left(x, t-\tau_{1}\right)-b_{1} u_{2}\left(x, t-\tau_{2}\right)\right], \\
\frac{\partial}{\partial t} u_{2}(x, t)=d_{2} \frac{\partial^{2}}{\partial x^{2}} u_{2}(x, t)+r_{2} u_{2}(x, t)\left[1-b_{2} u_{1}\left(x, t-\tau_{3}\right)-a_{2} u_{2}\left(x, t-\tau_{4}\right)\right] .
\end{array}\right.
$$

Thus, it is worthwhile to further explore this topic for systems without either QM or QM*, and this constitutes the purpose of this paper. 
In order to focus on the mathematical ideas and for the sake of simplicity, we consider a reaction-diffusion system of two equations with discrete delays, that is,

$$
\left\{\begin{array}{l}
\frac{\partial}{\partial t} u_{1}(x, t)=d_{1} \frac{\partial^{2}}{\partial x^{2}} u_{1}(x, t)+f_{1}\left(u_{1}\left(x, t-\tau_{11}\right), u_{2}\left(x, t-\tau_{12}\right)\right), \\
\frac{\partial}{\partial t} u_{2}(x, t)=d_{2} \frac{\partial^{2}}{\partial x^{2}} u_{2}(x, t)+f_{2}\left(u_{1}\left(x, t-\tau_{21}\right), u_{2}\left(x, t-\tau_{22}\right)\right),
\end{array}\right.
$$

where $d_{i}>0, \tau_{i j} \geqslant 0, f_{i}: \mathbb{R}^{2} \rightarrow \mathbb{R}$ is a continuous function, $x \in(-\infty, \infty)$, and

(A1) $f_{i}(0,0)=f_{i}\left(k_{1}, k_{2}\right)=0$ for $i=1,2$.

(A2) There exist two positive constants $L_{1}>0$ and $L_{2}>0$ such that

$$
\begin{aligned}
& \left|f_{1}\left(\phi_{1}, \psi_{1}\right)-f_{1}\left(\phi_{2}, \psi_{2}\right)\right| \leqslant L_{1}\|\Phi-\Psi\|, \\
& \left|f_{2}\left(\phi_{1}, \psi_{1}\right)-f_{2}\left(\phi_{2 t}, \psi_{2}\right)\right| \leqslant L_{2}\|\Phi-\Psi\|
\end{aligned}
$$

for $\Phi=\left(\phi_{1}, \psi_{1}\right), \Psi=\left(\phi_{2}, \psi_{2}\right) \in C\left([-\tau, 0], \mathbb{R}^{2}\right)$ with $0 \leqslant \phi_{i}(s), \psi_{i}(s) \leqslant M_{i}, s \in[-\tau, 0]$, $M_{i}>k_{i}$ is positive constant, $i=1,2$.

Since the results of Huang and Zou [15], Ma [22] and Wu and Zou [33] do not apply to delayed reaction-diffusion systems (1.2) and (1.3), we must search for new techniques that can be applied to our delayed reaction-diffusion system (1.4), at least for (1.2) and (1.3). To overcome the difficulty, we propose two new conditions on the reaction terms, which are to be called the weak QM condition (WQM) and the weak QM* condition (WQM*), respectively:

(WQM) Two positive numbers exist $\beta_{1}>0, \beta_{2}>0$ such that

$$
\begin{aligned}
& f_{1}\left(\phi_{1}(s), \psi_{1}(s)\right)-f_{1}\left(\phi_{2}(s), \psi_{1}(s)\right)+\beta_{1}\left[\phi_{1}(0)-\phi_{2}(0)\right] \geqslant 0, \\
& f_{1}\left(\phi_{1}(s), \psi_{1}(s)\right)-f_{1}\left(\phi_{1}(s), \psi_{2}(s)\right) \leqslant 0, \\
& f_{2}\left(\phi_{1}(s), \psi_{1}(s)\right)-f_{2}\left(\phi_{1}(s), \psi_{2}(s)\right)+\beta_{2}\left[\psi_{1}(0)-\psi_{2}(0)\right] \geqslant 0, \\
& f_{2}\left(\phi_{1}(s), \psi_{1}(s)\right)-f_{2}\left(\phi_{2}(s), \psi_{1}(s)\right) \leqslant 0
\end{aligned}
$$

for $\phi_{1}(s), \phi_{2}(s), \psi_{1}(s), \psi_{2}(s) \in C([-\tau, 0], \mathbb{R})$ with

$$
0 \leqslant \phi_{2}(s) \leqslant \phi_{1}(s) \leqslant M_{1}, 0 \leqslant \psi_{2}(s) \leqslant \psi_{1}(s) \leqslant M_{2}, s \in[-\tau, 0] .
$$

(WQM ${ }^{*}$ ) Two positive numbers exist $\beta_{1}>0, \beta_{2}>0$ such that

$$
\begin{aligned}
& f_{1}\left(\phi_{1}(s), \psi_{1}(s)\right)-f_{1}\left(\phi_{2}(s), \psi_{1}(s)\right)+\beta_{1}\left[\phi_{1}(0)-\phi_{2}(0)\right] \geqslant 0, \\
& f_{1}\left(\phi_{1}(s), \psi_{1}(s)\right)-f_{1}\left(\phi_{1}(s), \psi_{2}(s)\right) \leqslant 0, \\
& f_{2}\left(\phi_{1}(s), \psi_{1}(s)\right)-f_{2}\left(\phi_{1}(s), \psi_{2}(s)\right)+\beta_{2}\left[\psi_{1}(0)-\psi_{2}(0)\right] \geqslant 0, \\
& f_{2}\left(\phi_{1}(s), \psi_{1}(s)\right)-f_{2}\left(\phi_{2}(s), \psi_{1}(s)\right) \leqslant 0
\end{aligned}
$$

for $\phi_{1}(s), \phi_{2}(s), \psi_{1}(s), \psi_{2}(s) \in C([-\tau, 0], \mathbb{R})$ with

(i) $0 \leqslant \phi_{2}(s) \leqslant \phi_{1}(s) \leqslant M_{1}, 0 \leqslant \psi_{2}(s) \leqslant \psi_{1}(s) \leqslant M_{2}, s \in[-\tau, 0]$;

(ii) $e^{\beta_{1} s}\left[\phi_{1}(s)-\phi_{2}(s)\right]$ and $e^{\beta_{2} s}\left[\psi_{1}(s)-\psi_{2}(s)\right]$ are non-decreasing in $s \in[-\tau, 0]$.

Since the nonlinear functions $f_{1}$ and $f_{2}$ in (1.4) have different monotonicity with respect to the first and second arguments in the first and second equations, respectively, following Pao [23] and $\mathrm{Ye}$ and $\mathrm{Li}$ [34], we introduce definitions of the upper and lower solutions, and a new crossiteration scheme, which are different from those defined in Huang and Zou [15], Ma [22] and $\mathrm{Wu}$ and Zou [33]. By using such a scheme, we will construct a subset in the Banach space $C\left(\mathbb{R}, \mathbb{R}^{2}\right)$ equipped with the exponential decay norm and reduce the existence of travelling wave solutions to the existence of an admissible pair of upper and lower solutions which are easy to construct in practice. As applications, we shall show that system (1.2) satisfies the condition (WQM) while system (1.3) satisfies (WQM*) and establish the existence of travelling wave solutions in both models. 
We remark that if $\tau_{1}=\tau_{2}=0$, then (1.2) reduces to the following Lotka-Volterra diffusion-competition system:

$$
\left\{\begin{array}{l}
\frac{\partial}{\partial t} u_{1}(x, t)=d_{1} \frac{\partial^{2}}{\partial x^{2}} u_{1}(x, t)+r_{1} u_{1}(x, t)\left[1-a_{1} u_{1}(x, t)-b_{1} u_{2}(x, t)\right], \\
\frac{\partial}{\partial t} u_{2}(x, t)=d_{2} \frac{\partial^{2}}{\partial x^{2}} u_{2}(x, t)+r_{2} u_{2}(x, t)\left[1-b_{2} u_{1}(x, t)-a_{2} u_{2}(x, t)\right],
\end{array}\right.
$$

where $u_{1}$ and $u_{2}$ represent the densities of two competitive species in a one-dimensional habitat having infinite length and $d_{1}, d_{2}, r_{1}, r_{2}, a_{1}, a_{2}, b_{1}, b_{2}$ are some positive constants. The existence of travelling wave solutions of (1.5) has been extensively studied in the literature (Conley and Gardner [5], Gardner [8], Gourley and Ruan [9], Kanel and Zhou [16], Kan-on [17], Tang and Fife [29] and van Vuuren [30]). This model has a trivial (no species) equilibrium $E_{0}=(0,0)$, two semitrivial (one species only) spatially homogeneous equilibria [9]

$$
E_{1}=\left(\frac{1}{a_{1}}, 0\right), E_{2}=\left(0, \frac{1}{a_{2}}\right)
$$

and a positive (two coexisting species) spatially homogeneous equilibrium

$$
E^{*}=\left(\frac{a_{2}-b_{1}}{a_{1} a_{2}-b_{1} b_{2}}, \frac{a_{1}-b_{2}}{a_{1} a_{2}-b_{1} b_{2}}\right)
$$

provided that $a_{1} a_{2} \neq b_{1} b_{2}$ and either (i) $a_{2}>b_{1}$ and $a_{1}>b_{2}$ or (ii) $a_{2}<b_{1}$ and $a_{1}<b_{2}$. By using phase space analysis for the ordinary differential equations, Tang and Fife [29] and van Vuuren [30] showed that (1.5) has travelling front solutions connecting the equilibria $E_{0}$ and $E^{*}$. Kanel and Zhou [16] further proved that (1.5) has travelling front solutions connecting the equilibria $E_{1}$ and $E^{*}$. Conley and Gardner [5] and Gardner [8] showed that (1.5) has travelling front solutions connecting the equilibria $E_{1}$ and $E_{2}$, where Conley index and degree theory methods have been developed. Other related results can be found in Gourley and Ruan [9], Hosono [11, 12], Kan-on [17], etc. We shall establish the existence of travelling waves in system (1.4), thus in systems (1.2) and (1.3), that connect the trivial equilibrium $E_{0}$ and the positive equilibrium $E^{*}$. Thus our results can be regarded as a generalization of the results of Tang and Fife [29] and van Vuuren [30] to the diffusion-competition models with delays.

This paper is organized as following. Section 2 is devoted to some preliminary discussions. In section 3, we establish a new cross-iteration scheme and apply it to obtain the existence of travelling wave solutions if the nonlinear reaction term satisfies the condition (WQM). In section 4, we use the non-standard ordering of the profile set and prove that similar results hold if the nonlinear reaction term satisfies the condition (WQM*). In section 5, we apply our main results to the diffusion-competition systems (1.2) and (1.3) and prove the existence of travelling wave solutions. The paper ends with a discussion in section 6 .

\section{Preliminaries}

In this paper, we use the usual notations for the standard ordering in $\mathbb{R}^{2}$. That is, for $u=\left(u_{1}, u_{2}\right)$ and $v=\left(v_{1}, v_{2}\right)$, we denote $u \leqslant v$ if $u_{i} \leqslant v_{i}, i=1,2$, and $u<v$ if $u \leqslant v$ but $u \neq v$. In particular, we denote $u \ll v$ if $u \leqslant v$ but $u_{i} \neq v_{i}, i=1,2$. If $u \leqslant v$, we also denote $(u$, $v]=\left\{w \in \mathbb{R}^{2}, u<w \leqslant v\right\},[u, v)=\left\{w \in \mathbb{R}^{2}, u \leqslant w<v\right\}$, and $[u, v]=\left\{w \in \mathbb{R}^{2}\right.$, $u \leqslant w \leqslant v\}$. Let $|\cdot|$ denote the Euclidean norm in $\mathbb{R}^{2}$ and $\|\cdot\|$ denote the supremum norm in $C\left([-\tau, 0], \mathbb{R}^{2}\right)$.

A travelling wave solution of (1.4) is a special translation invariant solution of the form $u_{1}(x, t)=\phi(x+c t), u_{2}(x, t)=\psi(x+c t)$, where $(\phi, \psi) \in C^{2}\left(\mathbb{R}, \mathbb{R}^{2}\right)$ are the profiles of the wave that propagates through the one-dimensional spatial domain at a constant velocity 
$c>0$. Substituting $u_{1}(x, t)=\phi(x+c t), u_{2}(x, t)=\psi(x+c t)$ into (1.4) and denoting $\phi_{t}(s)=\phi(t+s), \psi_{t}(s)=\psi(t+s)$ and $x+c t$ by $t$, we find that (1.4) has a pair of travelling wave solutions if and only if the following wave equations

$$
\left\{\begin{array}{l}
d_{1} \phi^{\prime \prime}-c \phi^{\prime}+f_{1}^{c}\left(\phi_{t}, \psi_{t}\right)=0, \\
d_{2} \psi^{\prime \prime}-c \psi^{\prime}+f_{2}^{c}\left(\phi_{t}, \psi_{t}\right)=0
\end{array}\right.
$$

with asymptotic boundary conditions

$$
\lim _{t \rightarrow-\infty} \phi(t)=\phi_{-}, \quad \lim _{t \rightarrow+\infty} \phi(t)=\phi_{+}, \quad \lim _{t \rightarrow-\infty} \psi(t)=\psi_{-}, \quad \lim _{t \rightarrow+\infty} \psi(t)=\psi_{+}
$$

have a pair of solutions $(\phi(t), \psi(t))$ on $\mathbb{R}$, where $f_{i}^{c}(\phi, \psi): C([-\tau, 0], \mathbb{R}) \rightarrow \mathbb{R}, i=1,2$, is given by

$$
f_{i}^{c}(\phi, \psi)=f_{i}\left(\phi^{c}, \psi^{c}\right), \phi^{c}(s)=\phi(c s), \psi^{c}(s)=\psi(c s), s \in[-\tau, 0],
$$

where $\tau=\max _{1 \leqslant i, j \leqslant 2}\left\{\tau_{i j}\right\},\left(\phi_{-}, \psi_{-}\right)$and $\left(\phi_{+}, \psi_{+}\right)$are two equilibria of (2.1).

Without loss of generality, we let $\phi_{-}=0, \phi_{+}=k_{1}>0, \psi_{-}=0$ and $\psi_{+}=k_{2}>0$. Then boundary conditions (2.2) become

$\lim _{t \rightarrow-\infty} \phi(t)=0, \quad \lim _{t \rightarrow+\infty} \phi(t)=k_{1}, \quad \lim _{t \rightarrow-\infty} \psi(t)=0, \quad \lim _{t \rightarrow+\infty} \psi(t)=k_{2}$.

Let

$C_{[\mathbf{0}, \mathbf{M}]}\left(\mathbb{R}, \mathbb{R}^{2}\right)=\left\{(\phi, \psi) \in C\left(\mathbb{R}, \mathbb{R}^{2}\right): 0 \leqslant \phi(s) \leqslant M_{1}, 0 \leqslant \psi(s) \leqslant M_{2}, s \in \mathbb{R}\right\}$.

Define the operator $H=\left(H_{1}, H_{2}\right): C_{[\mathbf{0}, \mathbf{M}]}\left(\mathbb{R}, \mathbb{R}^{2}\right) \rightarrow C\left(\mathbb{R}, \mathbb{R}^{2}\right)$ by

$$
\left\{\begin{array}{l}
H_{1}(\phi, \psi)(t)=f_{1}^{c}\left(\phi_{t}, \psi_{t}\right)+\beta_{1} \phi(t), \\
H_{2}(\phi, \psi)(t)=f_{2}^{c}\left(\phi_{t}, \psi_{t}\right)+\beta_{2} \psi(t) .
\end{array}\right.
$$

Then (2.1) can be rewritten as following:

$$
\left\{\begin{array}{l}
d_{1} \phi^{\prime \prime}(t)-c \phi^{\prime}(t)-\beta_{1} \phi(t)+H_{1}(\phi, \psi)(t)=0 \\
d_{2} \psi^{\prime \prime}(t)-c \psi^{\prime}(t)-\beta_{2} \psi(t)+H_{2}(\phi, \psi)(t)=0
\end{array}\right.
$$

Let

$$
\begin{array}{ll}
\lambda_{1}=\frac{c-\sqrt{c^{2}+4 \beta_{1} d_{1}}}{2 d_{1}}, & \lambda_{2}=\frac{c+\sqrt{c^{2}+4 \beta_{1} d_{1}}}{2 d_{1}} \\
\lambda_{3}=\frac{c-\sqrt{c^{2}+4 \beta_{2} d_{2}}}{2 d_{2}}, & \lambda_{4}=\frac{c+\sqrt{c^{2}+4 \beta_{2} d_{2}}}{2 d_{2}} .
\end{array}
$$

Then

$$
\begin{aligned}
& \lambda_{1}<0<\lambda_{2}, \lambda_{3}<0<\lambda_{4}, \\
& d_{1} \lambda_{i}^{2}-c \lambda_{i}-\beta_{1}=0, i=1,2 \text { and } d_{2} \lambda_{i}^{2}-c \lambda_{i}-\beta_{2}=0, i=3,4 .
\end{aligned}
$$

Define the operator $F=\left(F_{1}, F_{2}\right): C_{[\mathbf{0}, \mathbf{M}]}\left(\mathbb{R}, \mathbb{R}^{2}\right) \rightarrow C\left(\mathbb{R}, \mathbb{R}^{2}\right)$ by

$$
\left\{\begin{array}{l}
F_{1}(\phi, \psi)(t)=\frac{1}{d_{1}\left(\lambda_{2}-\lambda_{1}\right)}\left[\int_{-\infty}^{t} e^{\lambda_{1}(t-s)} H_{1}(\phi, \psi)(s) \mathrm{d} s+\int_{t}^{\infty} e^{\lambda_{2}(t-s)} H_{1}(\phi, \psi)(s) \mathrm{d} s\right], \\
F_{2}(\phi, \psi)(t)=\frac{1}{d_{2}\left(\lambda_{4}-\lambda_{3}\right)}\left[\int_{-\infty}^{t} e^{\lambda_{3}(t-s)} H_{2}(\phi, \psi)(s) \mathrm{d} s+\int_{t}^{\infty} e^{\lambda_{4}(t-s)} H_{2}(\phi, \psi)(s) \mathrm{d} s\right] .
\end{array}\right.
$$

We can see that the operator $F$ is well defined and for any $(\phi, \psi) \in C_{[\mathbf{0}, \mathbf{M}]}\left(\mathbb{R}, \mathbb{R}^{2}\right)$,

$\left\{\begin{array}{l}d_{1}\left(F_{1}(\phi, \psi)\right)^{\prime \prime}(t)-c\left(F_{1}(\phi, \psi)\right)^{\prime}(t)-\beta_{1} F_{1}(\phi, \psi)(t)+H_{1}(\phi, \psi)(t)=0, \\ d_{2}\left(F_{2}(\phi, \psi)\right)^{\prime \prime}(t)-c\left(F_{2}(\phi, \psi)\right)^{\prime}(t)-\beta_{2} F_{2}(\phi, \psi)(t)+H_{2}(\phi, \psi)(t)=0 .\end{array}\right.$ 
Thus, a fixed point of $F$ is a solution of (2.5), which is a travelling wave solution of (1.4) connecting $\mathbf{0}=(0,0)$ and $\mathbf{K}=\left(k_{1}, k_{2}\right)$ if it satisfies (2.3).

In the following, we introduce the exponential decay norm. Let $\mu>0$ such that $\mu<\min \left\{-\lambda_{1}, \lambda_{2},-\lambda_{3}, \lambda_{4}\right\}$. Define

$$
B_{\mu}\left(\mathbb{R}, \mathbb{R}^{2}\right)=\left\{\Phi \in C\left(\mathbb{R}, \mathbb{R}^{2}\right): \sup _{t \in \mathbb{R}}|\Phi(t)| e^{-\mu|t|}<\infty\right\}
$$

and

$$
|\Phi|_{\mu}=\sup _{t \in \mathbb{R}}|\Phi(t)| e^{-\mu|t|} .
$$

Then it is easy to check that $\left(B_{\mu}\left(\mathbb{R}, \mathbb{R}^{2}\right),|\cdot|_{\mu}\right)$ is a Banach space.

\section{The case (WQM)}

In this section, we consider the existence of travelling wave solutions of (2.1) when the delayed reaction terms $f_{1}$ and $f_{2}$ satisfy the condition (WQM).

We start our cross-iteration with a pair of upper and lower solutions of (2.1) defined as follows.

Definition 3.1. A pair of twice continuously differentiable functions $\bar{\Phi}=(\bar{\phi}, \bar{\psi})$ and $\underline{\Phi}=(\underline{\phi}, \underline{\psi}) \in C\left(\mathbb{R}, \mathbb{R}^{2}\right)$ are called an upper and a lower solution of (2.1), respectively, if $\bar{\Phi}$ and $\underline{\Phi}$ satisfy

$$
\left\{\begin{array}{l}
d_{1} \bar{\phi}^{\prime \prime}(t)-c \bar{\phi}^{\prime}(t)+f_{1}^{c}\left(\bar{\phi}_{t}, \underline{\psi}_{t}\right) \leqslant 0 \text { on } \mathbb{R}, \\
d_{2} \bar{\psi}^{\prime \prime}(t)-c \bar{\psi}^{\prime}(t)+f_{2}^{c}\left(\underline{\phi}_{t}, \bar{\psi}_{t}\right) \leqslant 0 \text { on } \mathbb{R}
\end{array}\right.
$$

and

$$
\left\{\begin{array}{l}
d_{1} \underline{\phi}^{\prime \prime}(t)-c \underline{\phi}^{\prime}(t)+f_{1}^{c}\left(\underline{\phi}, \bar{\psi}_{t}\right) \geqslant 0 \text { on } \mathbb{R}, \\
d_{2} \underline{\psi}^{\prime \prime}(t)-c \underline{\psi}^{\prime}(t)+f_{2}^{c}\left(\bar{\phi}_{t}, \underline{\psi}_{t}\right) \geqslant 0 \text { on } \mathbb{R} .
\end{array}\right.
$$

In what follows, we assume that an upper solution $\bar{\Phi}=(\bar{\phi}, \bar{\psi})$ and a lower solution $\underline{\Phi}=(\underline{\phi}, \underline{\psi})$ of $(2.1)$ are given so that

$(\overline{\mathbf{P 1}}) \overline{\mathbf{0}} \leqslant(\phi, \psi) \leqslant(\bar{\phi}, \bar{\psi}) \leqslant \mathbf{M}=\left(M_{1}, M_{2}\right) ;$

(P2) $\lim _{t \rightarrow-\infty}(\bar{\phi}, \bar{\psi})=\mathbf{0}, \lim _{t \rightarrow+\infty}(\phi, \psi)=\lim _{t \rightarrow+\infty}(\bar{\phi}, \bar{\psi})=\mathbf{K}=\left(k_{1}, k_{2}\right)$.

For the operator $H=\left(H_{1}, H_{2}\right)$ defined in section 2 , we have the following result.

Lemma 3.2. Assume that (WQM) holds. Then

$$
H_{1}\left(\phi_{2}, \psi_{1}\right)(t) \leqslant H_{1}\left(\phi_{1}, \psi_{2}\right)(t), \quad H_{2}\left(\phi_{1}, \psi_{2}\right)(t) \leqslant H_{2}\left(\phi_{2}, \psi_{1}\right)(t)
$$

for $\left(\phi_{i}, \psi_{i}\right) \in C_{[\mathbf{0}, \mathbf{M}]}\left(\mathbb{R}, \mathbb{R}^{2}\right)$ with $0 \leqslant \phi_{2}(s) \leqslant \phi_{1}(s) \leqslant M_{1}, 0 \leqslant \psi_{2}(s) \leqslant \psi_{1}(s) \leqslant$ $M_{2}, s \in \mathbb{R}$.

Proof. By (WQM), direct calculation shows that

$$
\begin{aligned}
& H_{1}\left(\phi_{1}, \psi_{2}\right)(t)-H_{1}\left(\phi_{2}, \psi_{1}\right)(t) \\
& =H_{1}\left(\phi_{1}, \psi_{2}\right)(t)-H_{1}\left(\phi_{2}, \psi_{2}\right)(t)+H_{1}\left(\phi_{2}, \psi_{2}\right)(t)-H_{1}\left(\phi_{2}, \psi_{1}\right)(t) \\
& \geqslant H_{1}\left(\phi_{1}, \psi_{2}\right)(t)-H_{1}\left(\phi_{2}, \psi_{2}\right)(t) \\
& =f_{1}^{c}\left(\phi_{1 t}, \psi_{1 t}\right)-f_{1}^{c}\left(\phi_{2 t}, \psi_{1 t}\right)+\beta_{1}\left(\phi_{1}(t)-\phi_{2}(t)\right) \\
& \geqslant 0, t \in \mathbb{R} .
\end{aligned}
$$

The inequality for $H_{2}$ can be established similarly. The proof is complete. 
As a direct consequence of lemma 3.2, the following lemma is true.

Lemma 3.3. Assume that (WQM) holds. Then

$$
F_{1}\left(\phi_{2}, \psi_{1}\right)(t) \leqslant F_{1}\left(\phi_{1}, \psi_{2}\right)(t), \quad F_{2}\left(\phi_{1}, \psi_{2}\right)(t) \leqslant F_{2}\left(\phi_{2}, \psi_{1}\right)(t)
$$

for $\left(\phi_{i}, \psi_{i}\right) \in C_{[\mathbf{0}, \mathbf{M}]}\left(\mathbb{R}, \mathbb{R}^{2}\right)$ with $0 \leqslant \phi_{2}(s) \leqslant \phi_{1}(s) \leqslant M_{1}, 0 \leqslant \psi_{2}(s) \leqslant \psi_{1}(s) \leqslant$ $M_{2}, s \in \mathbb{R}$.

Now, we assume that there exists an upper solution $\bar{\Phi}(t)=(\bar{\phi}, \bar{\psi})$ and a lower solution $\underline{\Phi}=(\phi, \psi)$ of (2.1) satisfying (P1) and (P2).

Define the following profile set.

$\Gamma([\underline{\phi}, \underline{\psi}],[\bar{\phi}, \bar{\psi}])=\left\{(\phi, \psi) \in C_{[\mathbf{0}, \mathbf{M}]}\left(\mathbb{R}, \mathbb{R}^{2}\right), \underline{\phi}(t) \leqslant \phi(t) \leqslant \bar{\phi}(t), \underline{\psi}(t)\right.$

$$
\leqslant \psi(t) \leqslant \bar{\psi}(t), t \in \mathbb{R}\} \text {. }
$$

Obviously, $\Gamma([\underline{\phi}, \underline{\psi}],[\bar{\phi}, \bar{\psi}])$ is non-empty.

Lemma 3.4. Assume that (A2) holds. Then

$$
F=\left(F_{1}, F_{2}\right): C_{[\mathbf{0}, \mathbf{M}]}\left(\mathbb{R}, \mathbb{R}^{2}\right) \rightarrow C\left(\mathbb{R}, \mathbb{R}^{2}\right)
$$

is continuous with respect to the norm $|\cdot|_{\mu}$ in $B_{\mu}\left(\mathbb{R}, \mathbb{R}^{2}\right)$.

Proof. We first prove that $H_{1}: C_{[\mathbf{0}, \mathbf{M}]}\left(\mathbb{R}, \mathbb{R}^{2}\right) \rightarrow C\left(\mathbb{R}, \mathbb{R}^{2}\right)$ is continuous with respect to the norm $|\cdot|_{\mu}$. If $\Phi=\left(\phi_{1}, \psi_{1}\right), \Psi=\left(\phi_{2}, \psi_{2}\right) \in C_{[\mathbf{0}, \mathbf{M}]}\left(\mathbb{R}, \mathbb{R}^{2}\right)$ satisfy

$$
|\Phi-\Psi|_{\mu}=\sup _{t \in \mathbb{R}}|\Phi(t)-\Psi(t)| e^{-\mu|t|}<\delta
$$

then

$$
\begin{aligned}
& \left|H_{1}\left(\phi_{1}, \psi_{1}\right)(t)-H_{1}\left(\phi_{2}, \psi_{2}\right)(t)\right| e^{-\mu|t|} \\
= & \left|f_{1}^{c}\left(\phi_{1 t}, \psi_{1 t}\right)(t)-f_{1}^{c}\left(\phi_{2 t}, \psi_{2 t}\right)(t)+\beta_{1}\left(\phi_{1}(t)-\phi_{2}(t)\right)\right| e^{-\mu|t|} \\
\leqslant & L_{1}\left\|\Phi_{t}-\Psi_{t}\right\|_{C\left([-c \tau, 0], \mathbb{R}^{2}\right)} e^{-\mu|t|}+\beta_{1}\left|\phi_{1}-\phi_{2}\right|_{\mu} \\
= & L_{1} \sup _{s \in[-c \tau, 0]}|\Phi(t+s)-\Psi(t+s)| e^{-\mu|t|}+\beta_{1}\left|\phi_{1}-\phi_{2}\right|_{\mu} \\
\leqslant & L_{1} \sup _{\theta \in \mathbb{R}}|\Phi(\theta)-\Psi(\theta)| e^{-\mu|\theta|} e^{\mu c \tau}+\beta_{1}|\Phi-\Psi|_{\mu} \\
\leqslant & \left(L_{1} e^{\mu c \tau}+\beta_{1}\right)|\Phi-\Psi|_{\mu} .
\end{aligned}
$$

For any fixed $\varepsilon>0$, let $\delta<\varepsilon /\left(L_{1} e^{\mu c \tau}+\beta_{1}\right)$. If $\Phi=\left(\phi_{1}, \psi_{1}\right), \Psi=\left(\phi_{2}, \psi_{2}\right) \in C_{[\mathbf{0}, \mathbf{M}]}\left(\mathbb{R}, \mathbb{R}^{2}\right)$ satisfy $|\Phi-\Psi|_{\mu}<\delta$, then

$$
\begin{aligned}
& \left|H_{1}\left(\phi_{1}, \psi_{1}\right)(t)-H_{1}\left(\phi_{2}, \psi_{2}\right)(t)\right| e^{-\mu|t|} \\
\leqslant & \left(L_{1} e^{\mu c \tau}+\beta_{1}\right)|\Phi-\Psi|_{\mu}<\varepsilon .
\end{aligned}
$$

Therefore, $\left|H_{1}\left(\phi_{1}, \psi_{1}\right)-H_{1}\left(\phi_{2}, \psi_{2}\right)\right|_{\mu} \leqslant \varepsilon$. That is, $H_{1}: C_{[\mathbf{0}, \mathbf{M}]}\left(\mathbb{R}, \mathbb{R}^{2}\right) \rightarrow C\left(\mathbb{R}, \mathbb{R}^{2}\right)$ is continuous with respect to the norm $|\cdot|_{\mu}$.

Now, we show that $F_{1}: C_{[\mathbf{0}, \mathbf{M}]}\left(\mathbb{R}, \mathbb{R}^{2}\right) \rightarrow C\left(\mathbb{R}, \mathbb{R}^{2}\right)$ is continuous with respect to the $\operatorname{norm}|\cdot|_{\mu}$. 
For $t \geqslant 0$, we find

$$
\begin{aligned}
& \left|F_{1}\left(\phi_{1}, \psi_{1}\right)(t)-F_{1}\left(\phi_{2}, \psi_{2}\right)(t)\right| \\
\leqslant & \mid \frac{1}{d_{1}\left(\lambda_{2}-\lambda_{1}\right)}\left[\int_{-\infty}^{t} e^{\lambda_{1}(t-s)}\left|H_{1}\left(\phi_{1}, \psi_{1}\right)(s)-H_{1}\left(\phi_{2}, \psi_{2}\right)(s)\right| \mathrm{d} s \mid\right. \\
& \left.+\int_{t}^{\infty} e^{\lambda_{2}(t-s)}\left|H_{1}\left(\phi_{1}, \psi_{1}\right)(s)-H_{1}\left(\phi_{2}, \psi_{2}\right)(s)\right| \mathrm{d} s\right] \\
= & \frac{1}{d_{1}\left(\lambda_{2}-\lambda_{1}\right)}\left[\int_{-\infty}^{t} e^{\lambda_{1}(t-s)+\mu|s|}\left|H_{1}\left(\phi_{1}, \psi_{1}\right)(s)-H_{1}\left(\phi_{2}, \psi_{2}\right)(s)\right| e^{-\mu|s|} \mathrm{d} s\right. \\
& \left.+\int_{t}^{\infty} e^{\lambda_{2}(t-s)+\mu|s|}\left|H_{1}\left(\phi_{1}, \psi_{1}\right)(s)-H_{1}\left(\phi_{2}, \psi_{2}\right)(s)\right| e^{-\mu|s|} \mathrm{d} s\right] \\
\leqslant & \frac{1}{d_{1}\left(\lambda_{2}-\lambda_{1}\right)}\left[\int_{0}^{t} e^{\lambda_{1}(t-s)+\mu s} \mathrm{~d} s+\int_{-\infty}^{0} e^{\lambda_{1}(t-s)-\mu s} \mathrm{~d} s\right. \\
& \left.+\int_{t}^{\infty} e^{\lambda_{2}(t-s)+\mu s} \mathrm{~d} s\right]\left|H_{1}\left(\phi_{1}, \psi_{1}\right)-H_{1}\left(\phi_{2}, \psi_{2}\right)\right|_{\mu} \\
= & \frac{1}{d_{1}\left(\lambda_{2}-\lambda_{1}\right)}\left[\frac{\lambda_{2}-\lambda_{1}}{\left(\mu-\lambda_{1}\right)\left(\lambda_{2}-\mu\right)} e^{\mu t}+\frac{2 \mu}{\lambda_{1}^{2}-\mu^{2}} e^{\lambda_{1} t}\right] \\
& \times\left|H_{1}\left(\phi_{1}, \psi_{1}\right)-H_{1}\left(\phi_{2}, \psi_{2}\right)\right|_{\mu} .
\end{aligned}
$$

Hence, we have

$$
\begin{aligned}
& \left|F_{1}\left(\phi_{1}, \psi_{1}\right)(t)-F_{1}\left(\phi_{2}, \psi_{2}\right)(t)\right| e^{-\mu|t|} \\
\leqslant & \frac{1}{d_{1}\left(\lambda_{2}-\lambda_{1}\right)}\left[\frac{\lambda_{2}-\lambda_{1}}{\left(\mu-\lambda_{1}\right)\left(\lambda_{2}-\mu\right)}+\frac{2 \mu}{\lambda_{1}^{2}-\mu^{2}} e^{\left(\lambda_{1}-\mu\right) t}\right] \\
& \times\left|H_{1}\left(\phi_{1}, \psi_{1}\right)-H_{1}\left(\phi_{2}, \psi_{2}\right)\right|_{\mu} \\
\leqslant & \frac{1}{d_{1}\left(\lambda_{2}-\lambda_{1}\right)}\left[\frac{\lambda_{2}-\lambda_{1}}{\left(\mu-\lambda_{1}\right)\left(\lambda_{2}-\mu\right)}+\frac{2 \mu}{\lambda_{1}^{2}-\mu^{2}}\right] \\
& \times\left|H_{1}\left(\phi_{1}, \psi_{1}\right)-H_{1}\left(\phi_{2}, \psi_{2}\right)\right|_{\mu} .
\end{aligned}
$$

Similarly, for $t \leqslant 0$, we have

$\left|F_{1}\left(\phi_{1}, \psi_{1}\right)(t)-F_{1}\left(\phi_{2}, \psi_{2}\right)(t)\right| e^{-\mu|t|}$

$\leqslant \frac{1}{d_{1}\left(\lambda_{2}-\lambda_{1}\right)}\left[\frac{\lambda_{2}-\lambda_{1}}{-\left(\mu+\lambda_{1}\right)\left(\lambda_{2}+\mu\right)}+\frac{2 \mu}{\lambda_{2}^{2}-\mu^{2}}\right]\left|H_{1}\left(\phi_{1}, \psi_{1}\right)-H_{1}\left(\phi_{2}, \psi_{2}\right)\right|_{\mu}$.

Thus, it follows from (3.3) and (3.4) that $F_{1}: C_{[\mathbf{0}, \mathbf{M}]}\left(\mathbb{R}, \mathbb{R}^{2}\right) \rightarrow C\left(\mathbb{R}, \mathbb{R}^{2}\right)$ is continuous with respect to the norm $|\cdot|_{\mu}$ in $B_{\mu}\left(\mathbb{R}, \mathbb{R}^{2}\right)$.

By using a similar argument as above, we can also prove that $F_{2}: C_{[\mathbf{0}, \mathbf{M}]}\left(\mathbb{R}, \mathbb{R}^{2}\right) \rightarrow$ $C\left(\mathbb{R}, \mathbb{R}^{2}\right)$ is continuous with respect to the norm $|\cdot|_{\mu}$ in $B_{\mu}\left(\mathbb{R}, \mathbb{R}^{2}\right)$. This completes the proof.

Lemma 3.5. Assume that (WQM) holds. Then

$$
F: \Gamma([\underline{\phi}, \underline{\psi}],[\bar{\phi}, \bar{\psi}]) \subset \Gamma([\underline{\phi}, \underline{\psi}],[\bar{\phi}, \bar{\psi}]) .
$$

Proof. For any $(\phi, \psi) \in \Gamma([\underline{\phi}, \underline{\psi}],[\bar{\phi}, \bar{\psi}])$, by lemma 3.3, it is easy to see that

$$
\left\{\begin{array}{l}
F_{1}(\phi, \bar{\psi}) \leqslant F_{1}(\phi, \psi) \leqslant F_{1}(\bar{\phi}, \psi) \\
F_{2}(\overline{\bar{\phi}}, \underline{\psi}) \leqslant F_{2}(\phi, \psi) \leqslant F_{2}(\underline{\phi}, \overline{\bar{\psi}}) .
\end{array}\right.
$$


Now, we only need to prove

$$
\left\{\begin{array}{l}
\underline{\phi} \leqslant F_{1}(\underline{\phi}, \bar{\psi}) \leqslant F_{1}(\bar{\phi}, \underline{\psi}) \leqslant \bar{\phi}, \\
\underline{\psi} \leqslant F_{2}(\overline{\bar{\phi}}, \underline{\psi}) \leqslant F_{2}(\underline{\phi}, \bar{\psi}) \leqslant \bar{\psi} .
\end{array}\right.
$$

According to the definition of the operator $F$ and the lower solution, we have

$$
\begin{aligned}
F_{1}(\underline{\phi}, \bar{\psi})(t) & =\left[\int_{-\infty}^{t} e^{\lambda_{1}(t-s)}+\int_{-\infty}^{t} e^{\lambda_{2}(t-s)}\right] H_{1}(\underline{\phi}, \bar{\psi})(s) \mathrm{d} s \\
& \geqslant\left[\int_{-\infty}^{t} e^{\lambda_{1}(t-s)}+\int_{-\infty}^{t} e^{\lambda_{2}(t-s)}\right]\left(\beta_{1} \underline{\phi}(s)+c \underline{\phi^{\prime}}(s)-d_{1} \underline{\phi}^{\prime \prime}(s)\right) \mathrm{d} s \\
& =\underline{\phi}(t), t \in \mathbb{R} .
\end{aligned}
$$

In a similar way, we can prove that (3.5) holds for $t \in \mathbb{R}$. The proof is complete.

Lemma 3.6. Assume that (A2) and (WQM) hold. Then

$$
F: \Gamma([\underline{\phi}, \underline{\psi}],[\bar{\phi}, \bar{\psi}]) \rightarrow \Gamma([\underline{\phi}, \underline{\psi}],[\bar{\phi}, \bar{\psi}])
$$

is compact.

Proof. We first establish an estimate for $F$. For any $(\phi, \psi) \in \Gamma([\underline{\phi}, \underline{\psi}],[\bar{\phi}, \bar{\psi}])$, we have

$$
\begin{aligned}
\left(F_{1}(\phi, \psi)\right)^{\prime}(t)= & \frac{\lambda_{1} e^{\lambda_{1} t}}{d_{1}\left(\lambda_{2}-\lambda_{1}\right)} \int_{-\infty}^{t} e^{-\lambda_{1} s} H_{1}(\phi, \psi)(s) \mathrm{d} s \\
& +\frac{\lambda_{2} e^{\lambda_{2} t}}{d_{1}\left(\lambda_{2}-\lambda_{1}\right)} \int_{t}^{\infty} e^{-\lambda_{2} s} H_{1}(\phi, \psi)(s) \mathrm{d} s
\end{aligned}
$$

So

$$
\begin{aligned}
\left|\left(F_{1}(\phi, \psi)\right)^{\prime}\right|_{\mu} \leqslant & \sup _{t \in \mathbb{R}}\left[e^{-\mu|t|} \frac{\left|\lambda_{1}\right| e^{\lambda_{1} t}}{d_{1}\left(\lambda_{2}-\lambda_{1}\right)} \int_{-\infty}^{t} e^{-\lambda_{1} s} H_{1}(\phi, \psi)(s) \mathrm{d} s\right. \\
& \left.+e^{-\mu|t|} \frac{\lambda_{2} e^{\lambda_{2} t}}{d_{1}\left(\lambda_{2}-\lambda_{1}\right)} \int_{t}^{\infty} e^{-\lambda_{2} s} H_{1}(\phi, \psi)(s) \mathrm{d} s\right] \\
\leqslant & \frac{\left|\lambda_{1}\right|}{d_{1}\left(\lambda_{2}-\lambda_{1}\right)} \sup _{t \in \mathbb{R}} e^{\lambda_{1} t-\mu|t|} \int_{-\infty}^{t} e^{-\lambda_{1} s} e^{\mu|s|} e^{-\mu|s|} H_{1}(\phi, \psi)(s) \mathrm{d} s \\
& +\frac{\lambda_{2}}{d_{1}\left(\lambda_{2}-\lambda_{1}\right)} \sup _{t \in \mathbb{R}} e^{\lambda_{2} t-\mu|t|} \int_{t}^{\infty} e^{-\lambda_{2} s} e^{\mu|s|} e^{-\mu|s|} H_{1}(\phi, \psi)(s) \mathrm{d} s \\
\leqslant & \frac{\left|\lambda_{1}\right|}{d_{1}\left(\lambda_{2}-\lambda_{1}\right)}\left|H_{1}(\phi, \psi)\right|_{\mu} \sup _{t \in \mathbb{R}} e^{\lambda_{1} t-\mu|t|} \int_{-\infty}^{t} e^{-\lambda_{1} s+\mu|s|} \mathrm{d} s \\
& +\frac{\lambda_{2}}{d_{1}\left(\lambda_{2}-\lambda_{1}\right)}\left|H_{1}(\phi, \psi)\right|_{\mu} \sup _{t \in \mathbb{R}} e^{\lambda_{2} t-\mu|t|} \int_{t}^{\infty} e^{-\lambda_{2} s+\mu|s|} \mathrm{d} s .
\end{aligned}
$$

Therefore, for $t>0$, we have

$$
\begin{aligned}
\left|\left(F_{1}(\phi, \psi)\right)^{\prime}\right|_{\mu} \leqslant & \frac{\left|\lambda_{1}\right|}{d_{1}\left(\lambda_{2}-\lambda_{1}\right)\left(-\lambda_{1}-\mu\right)}\left|H_{1}(\phi, \psi)\right|_{\mu} \\
& +\frac{\lambda_{2}}{d_{1}\left(\lambda_{2}-\lambda_{1}\right)\left(\lambda_{2}-\mu\right)}\left|H_{1}(\phi, \psi)\right|_{\mu} \\
= & \frac{1}{d_{1}\left(\lambda_{2}-\lambda_{1}\right)}\left[\frac{\lambda_{1}}{\left(\lambda_{1}+\mu\right)}+\frac{\lambda_{2}}{\left(\lambda_{2}-\mu\right)}\right]\left|H_{1}(\phi, \psi)\right|_{\mu} .
\end{aligned}
$$


Similarly, for $t<0$, we have

$$
\begin{aligned}
\left|\left(F_{1}(\phi, \psi)\right)^{\prime}\right|_{\mu} \leqslant & \frac{\left|\lambda_{1}\right|}{d_{1}\left(\lambda_{2}-\lambda_{1}\right)\left(-\lambda_{1}-\mu\right)}\left|H_{1}(\phi, \psi)\right|_{\mu} \\
& +\frac{\lambda_{2}}{d_{1}\left(\lambda_{2}-\lambda_{1}\right)}\left[\left|\frac{1}{\left(\lambda_{2}-\mu\right)}-\frac{1}{\left(\lambda_{2}+\mu\right)}\right|+\frac{1}{\left(\lambda_{2}+\mu\right)}\right]\left|H_{1}(\phi, \psi)\right|_{\mu} \\
\leqslant & \frac{1}{d_{1}\left(\lambda_{2}-\lambda_{1}\right)}\left[\frac{\lambda_{1}}{\left(\lambda_{1}+\mu\right)}+\frac{\lambda_{2}}{\left(\lambda_{2}-\mu\right)}\right]\left|H_{1}(\phi, \psi)\right|_{\mu} .
\end{aligned}
$$

Since $H: C_{[\mathbf{0}, \mathbf{M}]}\left(\mathbb{R}, \mathbb{R}^{2}\right) \rightarrow C\left(\mathbb{R}, \mathbb{R}^{2}\right)$ is continuous with respect to the norm $|\cdot|_{\mu}$ and the set $\Gamma([\phi, \psi],[\bar{\phi}, \bar{\psi}])$ is uniformly bounded, there exists a constant $C_{1}$ such that $\left|\left(F_{1}(\phi, \psi)\right)^{\prime}\right|_{\mu} \leqslant$ $C_{1}$. In a similar way, there exists a constant $C_{2}$ such that $\left|\left(F_{2}(\phi, \psi)\right)^{\prime}\right|_{\mu} \leqslant C_{2}$. Hence $F$ is equicontinuous on $\Gamma([\phi, \psi],[\bar{\phi}, \bar{\psi}])$ and $F \Gamma([\phi, \psi],[\bar{\phi}, \bar{\psi}])$ is uniformly bounded.

We next prove that $F: \Gamma([\underline{\phi}, \underline{\psi}],[\bar{\phi}, \bar{\psi}]) \rightarrow \Gamma([\underline{\phi}, \underline{\psi}],[\bar{\phi}, \bar{\psi}])$ is compact.

Define $F^{n}(\phi, \psi)$ by

$$
F^{n}(\phi, \psi)(t)=\left\{\begin{array}{l}
F(\phi, \psi)(t), t \in[-n, n] ; \\
F(\phi, \psi)(n), t \in(n, \infty) ; \\
F(\phi, \psi)(-n), t \in(-\infty,-n) .
\end{array}\right.
$$

Then, for any $n \geqslant 1, F^{n}$ is equicontinuous and uniformly bounded. Ascoli-Arzela lemma implies that $F^{n}$ is compact.

Since $\left\{F^{n}(\phi, \psi)\right\}_{0}^{\infty}$ is a compact series, and

$$
\begin{aligned}
& \sup _{t \in \mathbb{R}}\left|F^{n}(\phi, \psi)(t)-F(\phi, \psi)(t)\right| e^{-\mu|t|} \\
= & \sup _{t \in(-\infty,-n) \cup(n, \infty)}\left|F^{n}(\phi, \psi)(t)-F(\phi, \psi)(t)\right| e^{-\mu|t|} \\
\leqslant & 2 C_{0} e^{-\mu n} \rightarrow 0 \text { as } n \rightarrow \infty,
\end{aligned}
$$

where $C_{0}$ is a positive constant such that

$$
|(\phi, \psi)| \leqslant C_{0} \text { for any }(\phi, \psi) \in \Gamma([\underline{\phi}, \underline{\psi}],[\bar{\phi}, \bar{\psi}])
$$

by proposition 2.1 in [35], we know that $\left\{F^{n}\right\}_{0}^{\infty}$ converges to $F$ in $\Gamma([\phi, \psi],[\bar{\phi}, \bar{\psi}])$ with respect to the norm $|\cdot|_{\mu}$. Therefore $F$ is compact.

Theorem 3.7. Assume that (A1), (A2) and (WQM) hold. If (2.1) has an upper solution $(\bar{\phi}, \bar{\psi}) \in C_{[\mathbf{0}, \mathbf{M}]}\left(\mathbb{R}, \mathbb{R}^{2}\right)$ and a lower solution $(\phi, \psi) \in C_{[\mathbf{0}, \mathbf{M}]}\left(\mathbb{R}, \mathbb{R}^{2}\right)$ such that $(P 1)$ and (P2) are satisfied, then (2.1) has a travelling wave solution satisfying (2.3).

Proof. From lemmas 3.4-3.6, we know that $F \Gamma([\underline{\phi}, \psi],[\bar{\phi}, \bar{\psi}]) \subset \Gamma([\underline{\phi}, \underline{\psi}],[\bar{\phi}, \bar{\psi}])$ and $F$ is compact. By Schauder's fixed point theorem there exists a fixed point $\left(\phi^{*}, \psi^{*}\right) \in$ $\Gamma([\underline{\phi}, \psi],[\bar{\phi}, \bar{\psi}])$, which is a solution of $(2.1)$.

In order to prove that this solution is a travelling wave solution, we need to verify the asymptotic boundary condition (2.3).

By $(\mathrm{P} 2)$ and the fact that

we see that

$$
0 \leqslant(\underline{\phi}(t), \underline{\psi}(t)) \leqslant\left(\phi^{*}(t), \psi^{*}(t)\right) \leqslant(\bar{\phi}(t), \bar{\psi}(t)) \leqslant\left(M_{1}, M_{2}\right),
$$

$$
\lim _{t \rightarrow-\infty}\left(\phi^{*}(t), \psi^{*}(t)\right)=(0,0) \text { and } \lim _{t \rightarrow \infty}\left(\phi^{*}(t), \psi^{*}(t)\right)=\left(k_{1}, k_{2}\right) .
$$

Therefore, the fixed point $\left(\phi^{*}(t), \psi^{*}(t)\right)$ satisfies the asymptotic boundary condition (2.3). The proof is complete. 
From theorem 3.7 we can see that the existence of solutions of (2.1) and (2.3) is reduced to the existence of an admissible pair of upper and lower solutions. However, it is difficult to construct such a pair of upper and lower solutions in practice because they are required to be twice continuously differentiable on $\mathbb{R}$.

In order to overcome the difficulty, we introduce the following weaker definitions of upper and lower solutions of (2.1) than definition 3.1. More precisely, we do not require that the upper and lower solutions are twice continuously differentiable on $\mathbb{R}$ and they are easy to construct in practice. See examples 5.1 and 5.5 in section 5 .

Definition 3.8. A pair of continuous functions $\bar{\Phi}=(\bar{\phi}, \bar{\psi}), \underline{\Phi}=(\underline{\phi}, \psi) \in C\left(\mathbb{R}, \mathbb{R}^{2}\right)$ is called a weak upper solution and a weak lower solution of (2.1), respectively, if constants $T_{i}, i=1, \cdots, m$ exist, such that $\bar{\Phi}$ and $\Phi$ are twice continuously differentiable in $\mathbb{R} \backslash\left\{T_{i}: i=1, \cdots, m\right\}$ and satisfy

$$
\left\{\begin{array}{l}
d_{1} \bar{\phi}^{\prime \prime}(t)-c \bar{\phi}^{\prime}(t)+f_{1}^{c}\left(\bar{\phi}_{t}, \psi_{t}\right) \leqslant 0, t \in \mathbb{R} \backslash\left\{T_{i}: i=1, \cdots, m\right\}, \\
d_{2} \bar{\psi}^{\prime \prime}(t)-c \bar{\psi}^{\prime}(t)+f_{2}^{c}\left(\underline{\phi}_{t}, \bar{\psi}_{t}\right) \leqslant 0, t \in \mathbb{R} \backslash\left\{T_{i}: i=1, \cdots, m\right\},
\end{array}\right.
$$

and

$$
\left\{\begin{array}{l}
d_{1} \underline{\phi}^{\prime \prime}(t)-c \underline{\phi}^{\prime}(t)+f_{1}^{c}\left(\underline{\phi}_{t}, \bar{\psi}_{t}\right) \geqslant 0, t \in \mathbb{R} \backslash\left\{T_{i}: i=1, \cdots, m\right\}, \\
d_{2} \underline{\psi}^{\prime \prime}(t)-c \underline{\psi}^{\prime}(t)+f_{2}^{c}\left(\bar{\phi}_{t}, \underline{\psi}_{t}\right) \geqslant 0, t \in \mathbb{R} \backslash\left\{T_{i}: i=1, \cdots, m\right\} .
\end{array}\right.
$$

Lemma 3.9. Assume that (WQM) holds. If $\bar{\Phi}, \underline{\Phi} \in C_{[\mathbf{0}, \mathbf{M}]}\left(\mathbb{R}, \mathbb{R}^{2}\right)$ are a weak upper solution and a weak lower solution of (2.1), respectively, satisfying (P1), (P2) and

$$
\left\{\begin{array}{l}
\bar{\phi}^{\prime}(t+) \leqslant \bar{\phi}^{\prime}(t-), \bar{\psi}^{\prime}(t+) \leqslant \bar{\psi}^{\prime}(t-), t \in \mathbb{R}, \\
\underline{\phi}^{\prime}(t+) \geqslant \underline{\phi^{\prime}}(t-), \underline{\psi^{\prime}}(t+) \geqslant \underline{\psi^{\prime}}(t-), t \in \mathbb{R},
\end{array}\right.
$$

then

$$
\underline{\Phi} \leqslant\left(F_{1}(\underline{\phi}, \bar{\psi}), F_{2}(\bar{\phi}, \underline{\psi})\right) \leqslant\left(F_{1}(\bar{\phi}, \underline{\psi}), F_{2}(\underline{\phi}, \bar{\psi})\right) \leqslant \bar{\Phi},
$$

and $\left(F_{1}(\phi, \bar{\psi}), F_{2}(\bar{\phi}, \psi)\right),\left(F_{1}(\bar{\phi}, \psi), F_{2}(\phi, \bar{\psi})\right) \in C_{[\mathbf{0}, \mathbf{M}]}\left(\mathbb{R}, \mathbb{R}^{2}\right)$ are a lower and an upper solution of $(2.1)$, respectively.

Proof. Without loss of generality, we assume that $\bar{\Phi}$ and $\underline{\Phi}$ are twice continuously differentiable in $\mathbb{R} \backslash\left\{T_{i}: i=1, \cdots, m\right\}$ with $-\infty<T_{1}<T_{2}<\cdots<T_{m}<+\infty$. Denote $T_{0}=-\infty$ and $T_{m+1}=+\infty$. It is easy to verify that $\left(F_{1}(\phi, \bar{\psi}), F_{2}(\bar{\phi}, \psi)\right)$ is well defined and twice continuously differentiable. For any $t \in\left(T_{k}, T_{k+1}\right), 0 \leqslant k \leqslant \bar{m}$, it follows from (2.6) and the definition of weak lower solution that

$$
\begin{aligned}
F_{1}(\underline{\phi}, \bar{\psi})(t)= & \frac{1}{d_{1}\left(\lambda_{2}-\lambda_{1}\right)}\left[\int_{-\infty}^{t} e^{\lambda_{1}(t-s)}+\int_{t}^{\infty} e^{\lambda_{2}(t-s)}\right] H_{1}(\underline{\phi}, \bar{\psi})(s) \mathrm{d} s \\
\geqslant & \frac{1}{d_{1}\left(\lambda_{2}-\lambda_{1}\right)}\left[\int_{-\infty}^{t} e^{\lambda_{1}(t-s)}+\int_{t}^{\infty} e^{\lambda_{2}(t-s)}\right] \\
& \times\left(\beta_{1} \underline{\phi}(s)+c \underline{\phi^{\prime}}(s)-d_{1} \underline{\phi}^{\prime \prime}(s)\right) \mathrm{d} s \\
= & \underline{\phi}(t)+\frac{1}{\lambda_{2}-\lambda_{1}}\left[\sum_{j=1}^{k} e^{\lambda_{2}\left(t-T_{i}\right)}\left(\underline{\phi}^{\prime}\left(T_{j}+\right)-\underline{\phi}^{\prime}\left(T_{j}-\right)\right)\right] \\
& +\frac{1}{\lambda_{2}-\lambda_{1}}\left[\sum_{j=k+1}^{m} e^{\lambda_{1}\left(t-T_{i}\right)}\left(\underline{\phi^{\prime}}\left(T_{j}+\right)-\underline{\phi^{\prime}}\left(T_{j}-\right)\right)\right] \\
\geqslant & \underline{\phi}(t) .
\end{aligned}
$$


Using a similar argument, we can prove that (3.9) holds. Furthermore, (2.7) together with lemma 3.2 yields

$$
\begin{aligned}
0= & d_{1}\left(F_{1}(\underline{\phi}, \bar{\psi})\right)^{\prime \prime}(t)-c\left(F_{1}(\underline{\phi}, \bar{\psi})\right)^{\prime}(t)-\beta_{1} F_{1}(\underline{\phi}, \bar{\psi})(t)+H_{1}(\underline{\phi}, \bar{\psi})(t) \\
\leqslant & d_{1}\left(F_{1}(\underline{\phi}, \bar{\psi})\right)^{\prime \prime}(t)-c\left(F_{1}(\underline{\phi}, \bar{\psi})\right)^{\prime}(t)-\beta_{1} F_{1}(\underline{\phi}, \bar{\psi})(t) \\
& +H_{1}\left(F_{1}(\underline{\phi}, \bar{\psi}), F_{2}(\underline{\phi}, \bar{\psi})\right)(t) \\
= & d_{1}\left(F_{1}(\underline{\phi}, \bar{\psi})\right)^{\prime \prime}(t)-c\left(F_{1}(\underline{\phi}, \bar{\psi})\right)^{\prime}(t)+f_{1}^{c}\left(F_{1}(\underline{\phi}, \bar{\psi}), F_{2}(\underline{\phi}, \bar{\psi})\right) .
\end{aligned}
$$

Similarly, we have

$$
d_{2}\left(F_{2}(\bar{\phi}, \underline{\psi})\right)^{\prime \prime}(t)-c\left(F_{2}(\bar{\phi}, \underline{\psi})\right)^{\prime}(t)+f_{2}^{c}\left(F_{1}(\bar{\phi}, \underline{\psi}), F_{2}(\bar{\phi}, \underline{\psi})\right) \geqslant 0 .
$$

Note that $\left(F_{1}(\phi, \bar{\psi}), F_{2}(\bar{\phi}, \psi)\right) \in C_{[\mathbf{0}, M]}\left(\mathbb{R}, \mathbb{R}^{2}\right) \cap C^{2}\left(\mathbb{R}, \mathbb{R}^{2}\right)$; we conclude that it is a lower solution of $(2 . \overline{1})$.

In a similar way, we can prove that $\left(F_{1}(\bar{\phi}, \psi), F_{2}(\phi, \bar{\psi})\right)$ is an upper solution of (2.1). The proof is complete.

Theorem 3.10. Assume that (A1), (A2) and (WQM) hold. If (2.1) has a weak upper solution $(\bar{\phi}, \bar{\psi})$ and a weak lower solution $(\phi, \psi)$ satisfying $(P 1),(P 2)$ and (3.8), then (2.1) has a travelling wave solution satisfying (2.3).

\section{The Case (WQM*)}

In this section, we shall consider the existence of travelling wave solutions of (2.1) when the delayed reaction terms $f_{1}$ and $f_{2}$ satisfy the condition (WQM ${ }^{*}$ ).

In what follows, we assume that an upper solution $(\bar{\phi}(t), \bar{\psi}(t))$ and a lower solution $(\underline{\phi}(t), \underline{\psi}(t))$ satisfy $(\mathrm{P} 1),(\mathrm{P} 2)$, and

(P) $e^{\beta_{1} s}[\bar{\phi}(s)-\phi(s)]$ and $e^{\beta_{2} s}[\bar{\psi}(s)-\psi(s)]$ are non-decreasing for $s \in \mathbb{R}$.

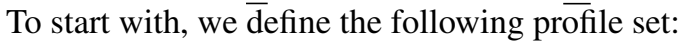

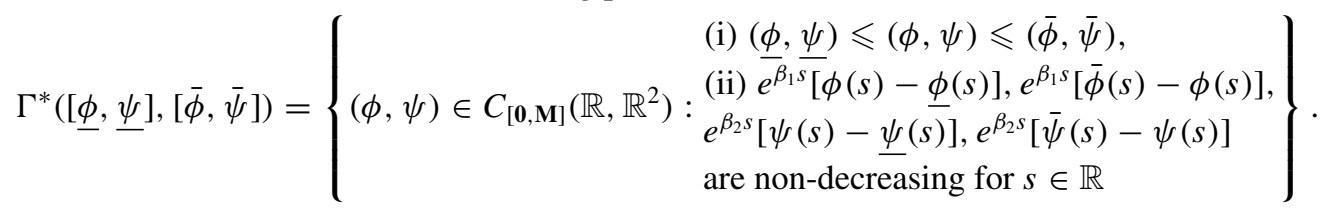

It is easy to see that $\Gamma^{*}([\phi, \psi],[\bar{\phi}, \bar{\psi}])$ is non-empty. In fact, by (P3), we know that $e^{\beta_{1} s}[\bar{\phi}(s)-\phi(s)]$ and $e^{\beta_{2} s}[\overline{\bar{\psi}}(s)-\psi(s)]$ are non-decreasing in $s \in \mathbb{R}, e^{\beta_{1} s}[\bar{\phi}(s)-\bar{\phi}(s)]=0$, and $e^{\beta_{2} s}[\bar{\psi}(\bar{s})-\bar{\psi}(s)]=0$. Thus $(\bar{\phi}, \bar{\psi})$ satisfies (ii) of $\Gamma^{*}([\underline{\phi}, \underline{\psi}],[\bar{\phi}, \bar{\psi}])$. Similarly, $(\underline{\phi}(t), \underline{\psi}(t))$ satisfies (ii) of $\Gamma^{*}([\underline{\phi}, \underline{\psi}],[\bar{\phi}, \bar{\psi}])$.

Lemma 4.1. Assume that $\left(W Q M^{*}\right)$ holds. Then

$$
H_{1}\left(\phi_{2}, \psi_{1}\right)(t) \leqslant H_{1}\left(\phi_{1}, \psi_{2}\right)(t), \quad H_{2}\left(\phi_{1}, \psi_{2}\right)(t) \leqslant H_{2}\left(\phi_{2}, \psi_{1}\right)(t),
$$

where $\Phi=\left(\phi_{1}, \psi_{1}\right), \Psi=\left(\phi_{2}, \psi_{2}\right) \in C_{[\mathbf{0}, \mathbf{M}]}\left(\mathbb{R}, \mathbb{R}^{2}\right)$ with $(i) 0 \leqslant \phi_{2}(s) \leqslant \phi_{1}(s) \leqslant M_{1}, 0 \leqslant$ $\psi_{2}(s) \leqslant \psi_{1}(s) \leqslant M_{2} ;$ (ii) $e^{\beta_{1} s}\left[\phi_{1}(s)-\phi_{2}(s)\right]$ and $e^{\beta_{2} s}\left[\psi_{1}(s)-\psi_{2}(s)\right]$ are non-decreasing for $s \in \mathbb{R}$.

Lemma 4.2. Assume that $\left(W Q M^{*}\right)$ holds. Then

$$
F_{1}\left(\phi_{2}, \psi_{1}\right)(t) \leqslant F_{1}\left(\phi_{1}, \psi_{2}\right)(t), \quad F_{2}\left(\phi_{1}, \psi_{2}\right)(t) \leqslant F_{2}\left(\phi_{2}, \psi_{1}\right)(t),
$$

where $\Phi=\left(\phi_{1}, \psi_{1}\right), \Psi=\left(\phi_{2}, \psi_{2}\right) \in C_{[\mathbf{0}, \mathbf{M}]}\left(\mathbb{R}, \mathbb{R}^{2}\right)$ with $(i) 0 \leqslant \phi_{2}(s) \leqslant \phi_{1}(s) \leqslant M_{1}, 0 \leqslant$ $\psi_{2}(s) \leqslant \psi_{1}(s) \leqslant M_{2} ;$ (ii) $e^{\beta_{1} s}\left[\phi_{1}(s)-\phi_{2}(s)\right]$ and $e^{\beta_{2} s}\left[\psi_{1}(s)-\psi_{2}(s)\right]$ are non-decreasing for $s \in \mathbb{R}$. 
Lemma 4.3. Assume that (A2) and $\left(W Q M^{*}\right)$ hold. Then $F: C_{[\mathbf{0}, \mathbf{M}]}\left(\mathbb{R}, \mathbb{R}^{2}\right) \rightarrow C\left(\mathbb{R}, \mathbb{R}^{2}\right)$ is continuous with respect to the norm $|\cdot|_{\mu}$ in $B_{\mu}\left(\mathbb{R}, \mathbb{R}^{2}\right)$.

The proofs of lemmas 4.1-4.3 are similar to those of lemmas 3.2-3.4 and are omitted here. By (P3), it is easy to see the following lemma.

Lemma 4.4. $\Gamma^{*}([\underline{\phi}, \underline{\psi}],[\bar{\phi}, \bar{\psi}])$ is a closed, bounded and convex subset of $B_{\mu}\left(\mathbb{R}, \mathbb{R}^{2}\right)$.

Lemma 4.5. Assume that (WQM*) holds. If $c>1-\min \left\{\beta_{1} d_{1}, \beta_{2} d_{2}\right\}$, then $F \Gamma^{*}([\underline{\phi}, \underline{\psi}],[\bar{\phi}, \bar{\psi}]) \subset \Gamma^{*}([\underline{\phi}, \underline{\psi}],[\bar{\phi}, \bar{\psi}])$.

Proof. For any $(\phi, \psi) \in \Gamma^{*}([\underline{\phi}, \underline{\psi}],[\bar{\phi}, \bar{\psi}])$, repeating the argument in the proof of lemma 3.5, we have

$$
\underline{\phi} \leqslant F_{1}(\phi, \psi) \leqslant \bar{\phi} \text { and } \underline{\psi} \leqslant F_{2}(\phi, \psi) \leqslant \bar{\psi} .
$$

This implies that (i) of $\Gamma^{*}([\underline{\phi}, \underline{\psi}],[\bar{\phi}, \bar{\psi}])$ holds. We now prove (ii) of $\Gamma^{*}([\underline{\phi}, \underline{\psi}],[\bar{\phi}, \bar{\psi}])$. Let $F_{1}(\phi, \psi)=\phi_{1}$ for $(\phi, \psi) \bar{\epsilon} \bar{\Gamma}^{*}$, then

$$
\begin{aligned}
& e^{\beta_{1} t}\left[\bar{\phi}(t)-\phi_{1}(t)\right] \\
= & \frac{e^{\beta_{1} t}}{d_{1}\left(\lambda_{2}-\lambda_{1}\right)}\left[\int_{-\infty}^{t} e^{\lambda_{1}(t-s)}+\int_{t}^{+\infty} e^{\lambda_{2}(t-s)}\right] \\
& \times\left\{\left[\beta_{1} \bar{\phi}(s)+c \bar{\phi}^{\prime}(s)-d_{1} \bar{\phi}^{\prime \prime}(s)\right]-\left[\beta_{1} \phi_{1}(s)+c \phi_{1}^{\prime}(s)-d_{1} \phi_{1}^{\prime \prime}(s)\right]\right\} \mathrm{d} s \\
= & \frac{e^{\beta_{1} t}}{d_{1}\left(\lambda_{2}-\lambda_{1}\right)}\left[\int_{-\infty}^{t} e^{\lambda_{1}(t-s)}+\int_{t}^{+\infty} e^{\lambda_{2}(t-s)}\right] \\
& \times\left\{\left[\beta_{1} \bar{\phi}(s)+c \bar{\phi}^{\prime}(s)-d_{1} \bar{\phi}^{\prime \prime}(s)-H_{1}(\phi, \psi)(s)\right]\right. \\
& \left.-\left[\beta_{1} \phi_{1}(s)+c \phi_{1}^{\prime}(s)-d_{1} \phi_{1}^{\prime \prime}(s)-H_{1}(\phi, \psi)(s)\right]\right\} \mathrm{d} s \\
= & \frac{e^{\beta_{1} t}}{d_{1}\left(\lambda_{2}-\lambda_{1}\right)}\left[\int_{-\infty}^{t} e^{\lambda_{1}(t-s)}+\int_{t}^{+\infty} e^{\lambda_{2}(t-s)}\right] \\
& \times\left[\beta_{1} \bar{\phi}(s)+c \bar{\phi}^{\prime}(s)-d_{1} \bar{\phi}^{\prime \prime}(s)-H_{1}(\phi, \psi)(s)\right] \mathrm{d} s .
\end{aligned}
$$

Hence, we have

$$
\begin{aligned}
& \frac{\mathrm{d}}{\mathrm{d} t}\left\{e^{\beta_{1} t}\left[\bar{\phi}(t)-\phi_{1}(t)\right]\right\} \\
= & \frac{\left(\beta_{1}+\lambda_{1}\right) e^{\beta_{1} t}}{d_{1}\left(\lambda_{2}-\lambda_{1}\right)} \int_{-\infty}^{t} e^{\lambda_{1}(t-s)}\left[\beta_{1} \bar{\phi}(s)+c \bar{\phi}^{\prime}(s)-d_{1} \bar{\phi}^{\prime \prime}(s)-H_{1}(\phi, \psi)(s)\right] \mathrm{d} s \\
& +\frac{\left(\beta_{1}+\lambda_{2}\right) e^{\beta_{1} t}}{d_{1}\left(\lambda_{2}-\lambda_{1}\right)} \int_{t}^{+\infty} e^{\lambda_{2}(t-s)}\left[\beta_{1} \bar{\phi}(s)+c \bar{\phi}^{\prime}(s)-d_{1} \bar{\phi}^{\prime \prime}(s)-H_{1}(\phi, \psi)(s)\right] \mathrm{d} s \\
\geqslant & \frac{\left(\beta_{1}+\lambda_{1}\right) e^{\beta_{1} t}}{d_{1}\left(\lambda_{2}-\lambda_{1}\right)} \int_{-\infty}^{t} e^{\lambda_{1}(t-s)}\left[\beta_{1} \bar{\phi}(s)+c \bar{\phi}^{\prime}(s)-d_{1} \bar{\phi}^{\prime \prime}(s)-H_{1}(\bar{\phi}, \underline{\psi})(s)\right] \mathrm{d} s \\
& +\frac{\left(\beta_{1}+\lambda_{2}\right) e^{\beta_{1} t}}{d_{1}\left(\lambda_{2}-\lambda_{1}\right)} \int_{t}^{+\infty} e^{\lambda_{2}(t-s)}\left[\beta_{1} \bar{\phi}(s)+c \bar{\phi}^{\prime}(s)-d_{1} \bar{\phi}^{\prime \prime}(s)-H_{1}(\bar{\phi}, \underline{\psi})(s)\right] \mathrm{d} s \\
\geqslant & 0, t \in \mathbb{R} .
\end{aligned}
$$

Similarly, we can prove that $e^{\beta_{2} t}\left[\bar{\psi}(t)-F_{2}(\phi, \psi)(t)\right], e^{\beta_{1} t}\left[F_{1}(\phi, \psi)(t)-\underline{\phi}(t)\right]$ and $e^{\beta_{2} t}\left[F_{2}(\phi, \psi)(t)-\underline{\psi}(t)\right]$ 
are non-decreasing in $t \in \mathbb{R}$. Thus, $F_{1}(\phi, \psi)$ satisfies (ii) of $\Gamma^{*}([\underline{\phi}, \underline{\psi}],[\bar{\phi}, \bar{\psi}])$. The proof is complete.

Similar to lemma 3.6 we have the following lemma.

Lemma 4.6. Assume that (A2) and $\left(W Q M^{*}\right)$ hold. Then

$$
F: \Gamma^{*}([\underline{\phi}, \underline{\psi}],[\bar{\phi}, \bar{\psi}]) \rightarrow \Gamma^{*}([\underline{\phi}, \underline{\psi}],[\bar{\phi}, \bar{\psi}])
$$

is compact.

Now we state our main result in this section; its proof is similar to that of theorem 3.7.

Theorem 4.7. Assume that (A1), (A2) and (WQM*) hold. Assume further that (2.1) has an upper solution $(\bar{\phi}, \bar{\psi}) \in C_{[\mathbf{0}, \mathbf{M}]}\left(\mathbb{R}, \mathbb{R}^{2}\right)$ and a lower $(\phi, \psi) \in C_{[\mathbf{0}, \mathbf{M}]}\left(\mathbb{R}, \mathbb{R}^{2}\right)$ satisfying $(P 1)$ (P3). Then, for any $c>1-\min \left\{\beta_{1} d_{1}, \beta_{2} d_{2}\right\}$, (2.1) has $\bar{a}$ travelling wave solution satisfying (2.3).

Similar to that in section 3, we have the following.

Theorem 4.8. Assume that (A1), (A2) and (WQM*) hold. If (2.1) has a weak upper solution $(\bar{\phi}, \bar{\psi}) \in C_{[\mathbf{0}, \mathbf{M}]}\left(\mathbb{R}, \mathbb{R}^{2}\right)$ and a weak lower solution $(\phi, \psi) \in C_{[\mathbf{0}, \mathbf{M}]}\left(\mathbb{R}, \mathbb{R}^{2}\right)$ such that $(P 1)$ (P3) and (3.8) hold, then, for any $c>1-\min \left\{\beta_{1} d_{1}, \beta_{2} \bar{d}_{2}\right\}$, (2.1) has a travelling wave solution satisfying (2.3).

Remark 4.9. If (WQM ${ }^{*}$ ) is satisfied, then we can always choose $\beta_{i}>0$ sufficiently large such that $c>1-\min \left\{\beta_{1} d_{1}, \beta_{2} d_{2}\right\}$.

\section{Applications}

As mentioned in the introduction, in this section, we employ our conclusions in sections 3 and 4 to establish the existence of travelling wave solutions for systems (1.2) and (1.3).

Example 5.1. We consider the existence of the travelling wave solutions for the delayed diffusion-competition system (1.2), that is,

$$
\left\{\begin{array}{l}
\frac{\partial}{\partial t} u_{1}(x, t)=d_{1} \frac{\partial^{2}}{\partial x^{2}} u_{1}(x, t)+r_{1} u_{1}(x, t)\left[1-a_{1} u_{1}(x, t)-b_{1} u_{2}\left(x, t-\tau_{1}\right)\right], \\
\frac{\partial}{\partial t} u_{2}(x, t)=d_{2} \frac{\partial^{2}}{\partial x^{2}} u_{2}(x, t)+r_{2} u_{2}(x, t)\left[1-b_{2} u_{1}\left(x, t-\tau_{2}\right)-a_{2} u_{2}(x, t)\right] .
\end{array}\right.
$$

Assume that $c>0$. Let $u_{1}(x, t)=u_{1}(x+c t)=\phi(s), u_{2}(x, t)=u_{2}(x+c t)=\psi(s)$, $s \in \mathbb{R}$, and denote the coordinate $s$ as $t$, then the corresponding wave system is

$$
\left\{\begin{array}{l}
d_{1} \phi^{\prime \prime}(t)-c \phi^{\prime}(t)+r_{1} \phi(t)\left[1-a_{1} \phi(t)-b_{1} \psi\left(t-c \tau_{1}\right)\right]=0, \\
d_{2} \psi^{\prime \prime}(t)-c \psi^{\prime}(t)+r_{2} \psi(t)\left[1-b_{2} \phi\left(t-c \tau_{2}\right)-a_{2} \psi(t)\right]=0 .
\end{array}\right.
$$

We are interested in solutions of (5.2) satisfying

$\lim _{t \rightarrow-\infty} \phi(t)=0, \quad \lim _{t \rightarrow+\infty} \phi(t)=k_{1}, \quad \lim _{t \rightarrow-\infty} \psi(t)=0, \quad \lim _{t \rightarrow+\infty} \psi(t)=k_{2}$,

where

$$
k_{1}=\frac{a_{2}-b_{1}}{a_{1} a_{2}-b_{1} b_{2}}>0, k_{2}=\frac{a_{1}-b_{2}}{a_{1} a_{2}-b_{1} b_{2}}>0,
$$

provided that

$$
a_{1}>b_{2}, a_{2}>b_{1} .
$$


For $\phi, \psi \in C([-\tau, 0], \mathbb{R})$, where $\tau=\max \left\{\tau_{1}, \tau_{2}\right\}$, denote

$$
\begin{aligned}
& f_{1}(\phi, \psi)=r_{1} \phi(0)\left[1-a_{1} \phi(0)-b_{1} \psi\left(-\tau_{1}\right)\right], \\
& f_{2}(\phi, \psi)=r_{2} \psi(0)\left[1-b_{2} \phi\left(-\tau_{2}\right)-a_{2} \psi(0)\right] .
\end{aligned}
$$

Obviously, (A1) and (A2) are satisfied. We now verify that $f=\left(f_{1}, f_{2}\right)$ satisfies (WQM).

Lemma 5.2. The function $f$ satisfies (WQM).

Proof. For any $\Phi(s)=\left(\phi_{1}(s), \phi_{2}(s)\right), \Psi(s)=\left(\psi_{1}(s), \psi_{2}(s)\right) \in C\left([-\tau, 0], \mathbb{R}^{2}\right)$ with $0 \leqslant \phi_{2}(s) \leqslant \phi_{1}(s) \leqslant M_{1}, 0 \leqslant \psi_{2}(s) \leqslant \psi_{1}(s) \leqslant M_{2}$, in view of $M_{1}>k_{1}$ and $M_{2}>k_{2}$, we have $2 a_{1} M_{1}+b_{1} M_{2}-1>2 a_{1} k_{1}+b_{1} k_{2}-1=a_{1} k_{1}>0$ and

$$
\begin{aligned}
& f_{1}\left(\phi_{1}, \psi_{1}\right)-f_{1}\left(\phi_{2}, \psi_{1}\right) \\
= & r_{1} \phi_{1}(0)\left[1-a_{1} \phi_{1}(0)-b_{1} \psi_{1}\left(-\tau_{1}\right)\right]-r_{1} \phi_{2}(0)\left[1-a_{1} \phi_{2}(0)-b_{1} \psi_{1}\left(-\tau_{1}\right)\right] \\
= & r_{1}\left[\phi_{1}(0)-\phi_{2}(0)\right]-r_{1} a_{1} \phi_{1}^{2}(0)-\phi_{2}^{2}(0)-r_{1} b_{1} \psi_{1}\left(-\tau_{1}\right)\left[\phi_{1}(0)-\phi_{2}(0)\right] \\
= & r_{1}\left[\phi_{1}(0)-\phi_{2}(0)\right]\left[1-a_{1}\left(\phi_{1}(0)+\phi_{2}(0)\right)-b_{1} \psi_{1}\left(-\tau_{1}\right)\right] \\
\geqslant & r_{1}\left[\phi_{1}(0)-\phi_{2}(0)\right]\left[1-2 a_{1} M_{1}-b_{1} M_{2}\right] \\
= & -r_{1}\left(2 a_{1} M_{1}+b_{1} M_{2}-1\right)\left[\phi_{1}(0)-\phi_{2}(0)\right] \\
= & -\beta_{1}\left[\phi_{1}(0)-\phi_{2}(0)\right]
\end{aligned}
$$

and

$$
\begin{aligned}
& f_{1}\left(\phi_{1}, \psi_{1}\right)-f_{1}\left(\phi_{1}, \psi_{2}\right) \\
= & r_{1} \phi_{1}(0)\left[1-a_{1} \phi_{1}(0)-b_{1} \psi_{1}\left(-\tau_{1}\right)\right]-r_{1} \phi_{1}(0)\left[1-a_{1} \phi_{1}(0)-b_{1} \psi_{2}\left(-\tau_{1}\right)\right] \\
= & -r_{1} b_{1} \phi_{1}(0)\left(\psi_{1}\left(-\tau_{1}\right)-\psi_{2}\left(-\tau_{1}\right)\right) \\
\leqslant & 0
\end{aligned}
$$

In a similar argument, we can prove that $f_{2}$ satisfies (WQM). The proof is complete.

In order to apply theorem 3.10, we need to construct a weak upper solution and a weak lower solution for (5.2).

If $c>\max \left\{2 \sqrt{d_{1} r_{1}}, 2 \sqrt{d_{2} r_{2}}\right\}$, then there exist $0<\lambda_{1}<\lambda_{2}$ such that

$$
d_{1} \lambda_{i}^{2}-c \lambda_{i}+r_{1}=0, i=1,2,
$$

and $0<\lambda_{3}<\lambda_{4}$ such that

$$
d_{2} \lambda_{i}^{2}-c \lambda_{i}+r_{2}=0, i=3,4 \text {. }
$$

For fixed

$$
\eta \in\left(1, \min \left\{2, \frac{\lambda_{2}}{\lambda_{1}}, \frac{\lambda_{4}}{\lambda_{3}}\right\}\right)
$$

and large constant $q>0$, we consider the functions $l_{1}(t)=e^{\lambda_{1} t}-q e^{\eta \lambda_{1} t}$ and $l_{2}(t)=$ $e^{\lambda_{3} t}-q e^{\eta \lambda_{3} t}$. It is easy to see that $l_{1}(t)$ and $l_{2}(t)$ have global maximum $m_{1}>0$ and $m_{2}>0$, respectively. Define

$$
t_{1}=\max \left\{t: l_{1}(t)=\frac{m_{1}}{2}\right\} \text { and } t_{3}=\max \left\{t: l_{2}(t)=\frac{m_{2}}{2}\right\} .
$$

Then for any given $\lambda>0$, there exists $\varepsilon_{2}>0$ and $\varepsilon_{4}>0$ such that

$$
k_{1}-\varepsilon_{2} e^{-\lambda t_{1}}=l_{1}\left(t_{1}\right)=\frac{m_{1}}{2} \text { and } k_{2}-\varepsilon_{4} e^{-\lambda t_{3}}=l_{2}\left(t_{3}\right)=\frac{m_{2}}{2} .
$$


Note that (5.3) holds, we have $\varepsilon_{0}>0, \varepsilon_{1}>0$ and $\varepsilon_{3}>0$ such that

$$
\left\{\begin{array}{l}
a_{1} \varepsilon_{1}-b_{1} \varepsilon_{4}>\varepsilon_{0}, a_{2} \varepsilon_{3}-b_{2} \varepsilon_{2}>\varepsilon_{0}, \\
a_{1} \varepsilon_{2}-b_{1} \varepsilon_{3}>\varepsilon_{0}, a_{2} \varepsilon_{4}-b_{2} \varepsilon_{1}>\varepsilon_{0} .
\end{array}\right.
$$

For the above constants and suitable constants $t_{2}, t_{4}$, we define the continuous functions as follows:

$$
\bar{\phi}(t)=\left\{\begin{array}{ll}
e^{\lambda_{1} t}, & t \leqslant t_{2}, \\
k_{1}+\varepsilon_{1} e^{-\lambda t}, & t \geqslant t_{2},
\end{array} \quad \bar{\psi}(t)= \begin{cases}e^{\lambda_{3} t}, & t \leqslant t_{4} \\
k_{2}+\varepsilon_{3} e^{-\lambda t}, & t \geqslant t_{4}\end{cases}\right.
$$

and

$$
\underline{\phi}(t)=\left\{\begin{array}{ll}
e^{\lambda_{1} t}-q e^{\eta \lambda_{1} t}, & t \leqslant t_{1}, \\
k_{1}-\varepsilon_{2} e^{-\lambda t}, & t \geqslant t_{1},
\end{array} \quad \underline{\psi}(t)= \begin{cases}e^{\lambda_{3} t}-q e^{\eta \lambda_{3} t}, & t \leqslant t_{3}, \\
k_{2}-\varepsilon_{4} e^{-\lambda t}, & t \geqslant t_{3},\end{cases}\right.
$$

where $q>0$ is large enough and $\lambda>0$ is small enough. It is easy to see $M_{1}=\sup _{t \in \mathbf{R}} \bar{\phi}(t)>$ $k_{1}, M_{2}=\sup _{t \in \mathbf{R}} \bar{\psi}(t)>k_{2}, \bar{\phi}(t), \bar{\psi}(t), \underline{\phi}(t)$ and $\psi(t)$ satisfy (P1), (P2), (3.8) and

$$
\min \left\{t_{2}, t_{4}\right\}-\max \left\{\tau_{1}, \tau_{2}\right\} \geqslant \max \left\{t_{1}, t_{3}\right\}
$$

for sufficiently large $q>0$ and sufficiently small $\lambda>0$. We now prove that the continuous functions $(\bar{\phi}(t), \bar{\psi}(t))$ and $(\phi(t), \psi(t))$ are a weak upper solution and a weak lower solution of (5.2), respectively.

Lemma 5.3. Assume that (5.3) and (5.5) hold. Then $\bar{\Phi}(t)=(\bar{\phi}(t), \bar{\psi}(t))$ is a weak upper solution and $\Phi(t)=(\phi(t), \psi(t))$ is a weak lower solution of (5.2).

Proof. For $t \leqslant t_{2}$, in view of $\psi\left(t-c \tau_{1}\right) \geqslant 0$ for $t \in \mathbb{R}$ and $d_{1} \lambda_{1}^{2}-c \lambda_{1}+r_{1}=0$, we have

$$
\begin{aligned}
& d_{1} \bar{\phi}^{\prime \prime}(t)-c \bar{\phi}^{\prime}(t)+r_{1} \bar{\phi}(t)\left[1-a_{1} \bar{\phi}(t)-b_{1} \underline{\psi}\left(t-c \tau_{1}\right)\right] \\
\leqslant & d_{1} \bar{\phi}^{\prime \prime}(t)-c \bar{\phi}^{\prime}(t)+r_{1} \bar{\phi}(t) \\
= & {\left[d_{1} \lambda_{1}^{2}-c \lambda_{1}+r_{1}\right]\left(k_{1}+\varepsilon_{1}\right) e^{\lambda_{1} t}=0 . }
\end{aligned}
$$

For $t \geqslant t_{2}$, since $\psi\left(t-c \tau_{1}\right)=k_{2}-\varepsilon_{4} e^{-\lambda\left(t-c \tau_{1}\right)}$, then

$$
\begin{aligned}
& d_{1} \bar{\phi}^{\prime \prime}(t)-c \bar{\phi}^{\prime}(t)+r_{1} \bar{\phi}(t)\left[1-a_{1} \bar{\phi}(t)-b_{1} \underline{\psi}\left(t-c \tau_{1}\right)\right] \\
= & e^{-\lambda t}\left\{d_{1} \varepsilon_{1} \lambda^{2}+c \varepsilon_{1} \lambda+r_{1}\left(k_{1}+\varepsilon_{1} e^{-\lambda t}\right)\left(b_{1} \varepsilon_{4} e^{\lambda c \tau_{1}}-a_{1} \varepsilon_{1}\right)\right\} .
\end{aligned}
$$

Let

$$
I_{1}(\lambda)=d_{1} \varepsilon_{1} \lambda^{2}+c \varepsilon_{1} \lambda+r_{1}\left(k_{1}+\varepsilon_{1} e^{-\lambda t}\right)\left(b_{1} \varepsilon_{4} e^{\lambda c \tau_{1}}-a_{1} \varepsilon_{1}\right) .
$$

Then, $a_{1} \varepsilon_{1}-b_{1} \varepsilon_{4}>\varepsilon_{0}$ implies that

$$
I_{1}(0)=r_{1}\left(k_{1}+\varepsilon_{1}\right)\left(b_{1} \varepsilon_{4}-a_{1} \varepsilon_{1}\right)<0,
$$

and there exists a $\lambda_{1}^{*}>0$ such that $I_{1}(\lambda)<0$ for $\lambda \in\left(0, \lambda_{1}^{*}\right)$. Thus, we have

$$
d_{1} \bar{\phi}^{\prime \prime}(t)-c \bar{\phi}^{\prime}(t)+r_{1} \bar{\phi}(t)\left[1-a_{1} \bar{\phi}(t)-b_{1} \underline{\psi}\left(t-c \tau_{1}\right)\right] \leqslant 0 .
$$

Similarly, there exists a $\lambda_{2}^{*}>0$ such that for $\lambda \in\left(0, \lambda_{2}^{*}\right)$ we have

$$
d_{2} \bar{\psi}^{\prime \prime}(t)-c \bar{\psi}^{\prime}(t)+r_{2} \bar{\psi}(t)\left[1-b_{2} \underline{\phi}\left(t-c \tau_{2}\right)-a_{2} \bar{\psi}(t)\right] \leqslant 0 .
$$

Taking $\lambda \in\left(0, \min \left(\lambda_{1}^{*}, \lambda_{2}^{*}\right)\right)$, we see that our conclusion is true.

A similar argument applies to $\underline{\Phi}(t)=(\phi(t), \psi(t))$. The proof is complete. 
By theorem 3.10, we have the following result.

Theorem 5.4. Assume that (5.3) holds. Then for every $c>\max \left\{2 \sqrt{d_{1} r_{1}}, 2 \sqrt{d_{2} r_{2}}\right\}$, (5.1) has a travelling wave solution $(\phi(x+c t), \psi(x+c t))$ with wave speed $c$, which connects $(0,0)$ and $\left(k_{1}, k_{2}\right)$. Furthermore, $\lim _{\xi \rightarrow-\infty}\left(\phi(\xi) e^{-\lambda_{1} \xi}, \psi(\xi) e^{-\lambda_{3} \xi}\right)=(1,1)$, where $\xi=x+c t$.

Example 5.5. We now consider the delayed diffusion-competition system (1.3), that is

$$
\left\{\begin{array}{l}
\frac{\partial}{\partial t} u_{1}(x, t)=d_{1} \frac{\partial^{2}}{\partial x^{2}} u_{1}(x, t)+r_{1} u_{1}(x, t)\left[1-a_{1} u_{1}\left(x, t-\tau_{1}\right)-b_{1} u_{2}\left(x, t-\tau_{2}\right)\right], \\
\frac{\partial}{\partial t} u_{2}(x, t)=d_{2} \frac{\partial^{2}}{\partial x^{2}} u_{2}(x, t)+r_{2} u_{2}(x, t)\left[1-b_{2} u_{1}\left(x, t-\tau_{3}\right)-a_{2} u_{2}\left(x, t-\tau_{4}\right)\right] .
\end{array}\right.
$$

The corresponding travelling wave system is

$$
\left\{\begin{array}{l}
d_{1} \phi^{\prime \prime}(t)-c \phi^{\prime}(t)+r_{1} \phi(t)\left[1-a_{1} \phi\left(t-c \tau_{1}\right)-b_{1} \psi\left(t-c \tau_{2}\right)\right]=0, \\
d_{2} \psi^{\prime \prime}(t)-c \psi^{\prime}(t)+r_{2} \psi(t)\left[1-b_{2} \phi\left(t-c \tau_{3}\right)-a_{2} \psi\left(t-c \tau_{4}\right)\right]=0 .
\end{array}\right.
$$

For $\phi, \psi \in C([-\tau, 0], \mathbb{R})$ with $\tau=\max \left\{\tau_{1}, \tau_{2}, \tau_{3}, \tau_{4}\right\}$, we denote

$$
\begin{aligned}
& f_{1}(\phi, \psi)=r_{1} \phi(0)\left[1-a_{1} \phi\left(-\tau_{1}\right)-b_{1} \psi\left(-\tau_{2}\right)\right], \\
& f_{2}(\phi, \psi)=r_{2} \psi(0)\left[1-b_{2} \phi\left(-\tau_{3}\right)-a_{2} \psi\left(-\tau_{4}\right)\right] .
\end{aligned}
$$

Obviously, (A1) and (A2) are satisfied. We now verify that $f=\left(f_{1}, f_{2}\right)$ satisfies (WQM*).

Lemma 5.6. Assume that $\tau_{1}$ and $\tau_{4}$ are small enough. Then the function $f$ satisfies $\left(W Q M^{*}\right)$.

Proof. For any $\Phi(s)=\left(\phi_{1}(s), \phi_{2}(s)\right), \Psi(s)=\left(\psi_{1}(s), \psi_{2}(s)\right) \in C\left([-\tau, 0], \mathbb{R}^{2}\right)$ with (i) $0 \leqslant \phi_{2}(s) \leqslant \phi_{1}(s) \leqslant M_{1}, 0 \leqslant \psi_{2}(s) \leqslant \psi_{1}(s) \leqslant M_{2}$ and (ii) $e^{\beta_{1} s}\left[\phi_{1}(s)-\phi_{2}(s)\right]$ and $e^{\beta_{2} s}\left[\psi_{1}(s)-\psi_{2}(s)\right]$ are non-decreasing in $s \in[-\tau, 0]$, we have

$$
\begin{aligned}
& f_{1}\left(\phi_{1}, \psi_{1}\right)-f_{1}\left(\phi_{2}, \psi_{1}\right) \\
= & r_{1} \phi_{1}(0)\left[1-a_{1} \phi_{1}\left(-\tau_{1}\right)-b_{1} \psi_{1}\left(-\tau_{2}\right)\right]-r_{1} \phi_{2}(0)\left[1-a_{1} \phi_{2}\left(-\tau_{1}\right)-b_{1} \psi_{1}\left(-\tau_{2}\right)\right] \\
= & r_{1}\left[\phi_{1}(0)-\phi_{2}(0)\right]-r_{1} a_{1}\left[\phi_{1}(0) \phi_{1}\left(-\tau_{1}\right)-\phi_{2}(0) \phi_{2}\left(-\tau_{1}\right)\right] \\
& -r_{1} b_{1} \psi_{1}\left(-\tau_{2}\right)\left[\phi_{1}(0)-\phi_{2}(0)\right] \\
\geqslant & \left(r_{1}-r_{1} b_{1} M_{2}\right)\left[\phi_{1}(0)-\phi_{2}(0)\right]-r_{1} a_{1} \phi_{1}(0)\left[\phi_{1}\left(-\tau_{1}\right)-\phi_{2}\left(-\tau_{1}\right)\right] \\
& -r_{1} a_{1} \phi_{2}\left(-\tau_{1}\right)\left[\phi_{1}(0)-\phi_{2}(0)\right] \\
\geqslant & r_{1}\left(1-b_{1} M_{2}-a_{1} M_{1}\right)\left[\phi_{1}(0)-\phi_{2}(0)\right] \\
& -r_{1} a_{1} \phi_{1}(0) e^{\beta_{1} \tau_{1}} e^{-\beta_{1} \tau_{1}}\left[\phi_{1}\left(-\tau_{1}\right)-\phi_{2}\left(-\tau_{1}\right)\right] \\
\geqslant & r_{1}\left(1-b_{1} M_{2}-a_{1} M_{1}-a_{1} M_{1} e^{\beta_{1} \tau_{1}}\right)\left[\phi_{1}(0)-\phi_{2}(0)\right] .
\end{aligned}
$$

If $\tau_{1}>0$ is small enough, then we can choose $\beta_{1}>0$ such that

$$
r_{1}\left(1-b_{1} M_{2}-a_{1} M_{1}-a_{1} M_{1} e^{\beta_{1} \tau_{1}}\right)>-\beta_{1} .
$$

We have

$$
\begin{aligned}
& f_{1}\left(\phi_{1}, \psi_{1}\right)-f_{1}\left(\phi_{1}, \psi_{2}\right) \\
= & r_{1} \phi_{1}(0)\left[1-a_{1} \phi_{1}\left(-\tau_{1}\right)-b_{1} \psi_{1}\left(-\tau_{2}\right)\right]-r_{1} \phi_{1}(0)\left[1-a_{1} \phi_{1}\left(-\tau_{1}\right)-b_{1} \psi_{2}\left(-\tau_{2}\right)\right] \\
= & -r_{1} b_{1} \phi_{1}(0)\left[\psi_{1}\left(-\tau_{2}\right)-\psi_{2}\left(-\tau_{2}\right)\right] \\
\leqslant & 0 .
\end{aligned}
$$

Thus $f_{1}(\phi, \psi)$ satisfies (WQM $\left.{ }^{*}\right)$ if $\tau_{1}$ is small enough.

In a similar way, we can prove that $f_{2}(\phi, \psi)$ satisfies (WQM $\left.{ }^{*}\right)$ if $\tau_{4}$ is small enough. The proof is complete. 
Remark 5.7. From the proof of lemma 5.6 we can see that if $\tau_{1}$ and $\tau_{4}$ are small enough, then we can always choose $\beta_{i}>0$ sufficiently large such that $c>1-\min \left\{\beta_{1} d_{1}, \beta_{2} d_{2}\right\}$.

Now we define $\bar{\phi}(t), \bar{\psi}(t), \underline{\phi(t)}$ and $\psi(t)$ as in example 5.1. It is easy to see that $\bar{\phi}(t), \bar{\psi}(t), \underline{\phi}(t)$ and $\underline{\psi}(t)$ satisfy $(\mathrm{P} 1)-(\mathrm{P} 3)$ and (3.8).

Lemma 5.8. Assume that (5.3) and (5.5) hold. If $\tau_{1}$ and $\tau_{4}$ are small enough, then $\bar{\Phi}(t)=$ $(\bar{\phi}(t), \bar{\psi}(t))$ is a weak upper solution and $\Phi(t)=(\phi(t), \psi(t))$ is a weak lower solution of (5.7).

Proof. For $\bar{\phi}(t)$, we need to prove that

$$
d_{1} \bar{\phi}^{\prime \prime}(t)-c \bar{\phi}^{\prime}(t)+r_{1} \bar{\phi}(t)\left[1-a_{1} \bar{\phi}\left(t-c \tau_{1}\right)-b_{1} \underline{\psi}\left(t-c \tau_{2}\right)\right] \leqslant 0 .
$$

For $t>t_{2}+c \tau_{1}$ or $t<t_{2}$, the proof of (5.8) is similar to that of lemma 5.3: we omit it here.

For $t_{2}<t<t_{2}+c \tau_{1}$, we note that we can choose $\lambda$ small enough such that

$$
d_{1} \bar{\phi}^{\prime \prime}(t)-c \bar{\phi}^{\prime}(t)+r_{1} \bar{\phi}(t)\left[1-a_{1} \bar{\phi}\left(t-c \tau_{1}\right)-b_{1} \underline{\psi}\left(t-c \tau_{2}\right)\right]<0,
$$

for $t=t_{2}+c \tau_{1}$. Since $\tau_{1}$ is small enough and independent of $\bar{\phi}, \psi$, and $\bar{\phi}^{\prime \prime}(t), \bar{\phi}^{\prime}(t), \bar{\phi}(t)$ and $\psi(t)$ are uniformly bounded and uniformly continuous for $t \in \overline{\mathbb{R} \backslash}\left\{t_{2}, t_{3}\right\}$, it follows that (5.8) holds for $t_{2}<t<t_{2}+c \tau_{1}$.

Similarly, we can prove that $\bar{\psi}$ satisfies

$$
d_{2} \bar{\psi}^{\prime \prime}(t)-c \bar{\psi}^{\prime}(t)+r_{2} \bar{\psi}(t)\left[1-b_{2} \underline{\phi}\left(t-c \tau_{3}\right)-a_{2} \bar{\psi}\left(t-c \tau_{4}\right)\right] \leqslant 0
$$

and $\underline{\Phi}(t)=(\underline{\phi}(t), \underline{\psi}(t))$ is a weak lower solution. The proof is complete.

Theorem 5.9. Assume that (5.3) holds and $\tau_{1}$ and $\tau_{4}$ are sufficiently small. Then for every $c>\max \left\{2 \sqrt{d_{1} r_{1}}, 2 \sqrt{d_{2} r_{2}}\right\}$, (5.6) has a travelling wave solution $(\phi(x+c t), \psi(x+c t))$ with wave speed $c$, which connects $(0,0)$ and $\left(k_{1}, k_{2}\right)$. Furthermore,

$$
\lim _{\xi \rightarrow-\infty}\left(\phi(\xi) e^{-\lambda_{1} \xi}, \psi(\xi) e^{-\lambda_{3} \xi}\right)=(1,1)
$$

where $\xi=x+c t$.

Remark 5.10. We note that the delay of example 5.1 does not affect the existence of travelling wave solutions. However, the delays $\left(\tau_{1}\right.$ and $\left.\tau_{4}\right)$ of example 5.5 do.

Remark 5.11. If $\tau=0$, then (5.6) reduces to (1.5), that is,

$$
\left\{\begin{array}{l}
\frac{\partial}{\partial t} u_{1}(x, t)=d_{1} \frac{\partial^{2}}{\partial x^{2}} u_{1}(x, t)+r_{1} u_{1}(x, t)\left[1-a_{1} u_{1}(x, t)-b_{1} u_{2}(x, t)\right], \\
\frac{\partial}{\partial t} u_{2}(x, t)=d_{2} \frac{\partial^{2}}{\partial x^{2}} u_{2}(x, t)+r_{2} u_{2}(x, t)\left[1-b_{2} u_{1}(x, t)-a_{2} u_{2}(x, t)\right] .
\end{array}\right.
$$

Tang and Fife [29] and van Vuuren [30] proved that (5.9) has a bounded travelling wave front solution connecting $(0,0)$ and $\left(k_{1}, k_{2}\right)$ if and only if $c \geqslant \max \left\{2 \sqrt{d_{1} r_{1}}, 2 \sqrt{d_{2} r_{2}}\right\}$. Our results certainly include their results when $c>\max \left\{2 \sqrt{d_{1} r_{1}}, 2 \sqrt{d_{2} r_{2}}\right\}$. If $c=\max \left\{2 \sqrt{d_{1} r_{1}}, 2 \sqrt{d_{2} r_{2}}\right\}$, we can get the existence of travelling wave solution only by changing the definition of $\phi$ and $\psi$. However, our results cannot ensure that the bounded travelling wave solutions of systems $(5 . \overline{1})$ and (5.6) connecting $(0,0)$ and $\left(k_{1}, k_{2}\right)$ are monotonic. Numerical simulations indicate that the travelling wave solutions are monotonic. It would be interesting to study the monotonicity of the travelling wave solutions in the delayed diffusion-competition models. 


\section{Discussion}

Coexistence of competing species is very common in natural systems (Durrett and Levin [6,7], Silvertown et al [28]). In the classical competition models (described by ordinary differential equations and used quantities averaged over space) spatial heterogeneity is usually neglected, while in real systems species are distributed spatially; organisms experience different local environments, they consume resources locally and move around. It is known now that spatial heterogeneity is crucial to the dynamics of biological systems, in particular for the coexistence of some competing species (Durrett and Levin [6]).

Different types of mathematical models have been used to describe spatial heterogeneous environments. For example, in metapopulation models (Hanski and Gilpin [10]) space is represented as a set of patches with local interactions. Reaction-diffusion equation models (Cantrell and Cosner [4]) take account of space explicitly. Stochastic spatial models (Durrett and Levin $[6,7])$ are a combination of these two approaches.

In this paper we considered a class of delayed reaction-diffusion systems without monotonicity. By using Schauder's fixed point theorem, a new cross-iteration scheme was given to establish the existence of travelling wave solutions. More precisely, by using such a new cross-iteration, we reduced the existence of travelling wave solutions to the existence of an admissible pair of upper and lower solutions which are easy to construct in practice. The general results were then applied to study the existence of travelling wave solutions in delayed two-species diffusion-competition systems. It is interesting to note that the delays appearing in the interspecific competition terms do not affect the existence of travelling waves while the delays appearing in the intraspecific competition terms do. The existence of travelling wave solutions which connect the trivial equilibrium $(0,0)$ and the positive equilibrium $\left(k_{1}, k_{2}\right)$ indicates that there is a transition zone moving from the steady state with no species to the steady state with the coexistence of both species.

It is also known that the dynamics of reactive systems on supports of restricted geometry may deviate substantially from the predictions of mean-field descriptions (Provata et al [24]). To couple microscope level processes and the evolution of the macroscope observables, nonlinear models on regular lattices have also been proposed (Kowalik et al [18], Provata et al [24], Rauch and Bar-Yam [25] and the references cited therein). The delayed lattice differential equations version of our model (1.4) takes the following form:

$$
\left\{\begin{array}{l}
\frac{d u_{n}}{d t}=\sum_{j=1}^{m} a_{j}\left[u_{n+j}(t)-2 u_{n}(t)+u_{n-j}(t)\right]+f_{1}\left(u_{n}\left(t-\tau_{11}\right), v_{n}\left(t-\tau_{12}\right)\right), \\
\frac{d v_{n}}{d t}=\sum_{j=1}^{m} b_{j}\left[v_{n+j}(t)-2 v_{n}(t)+v_{n-j}(t)\right]+f_{2}\left(u_{n}\left(t-\tau_{21}\right), v_{n}\left(t-\tau_{22}\right)\right),
\end{array}\right.
$$

where $n \in Z, m \geqslant 1$ is an integer, $a_{j}>0, b_{j}>0,1 \leqslant j \leqslant m, \tau \geqslant 0$. It would be interesting to combine the techniques and results in Huang et al [13] and the present paper to establish the existence of travelling waves for system (6.1) and apply the results to two species lattice competition models.

\section{Acknowledgments}

The authors are very grateful to an anonymous referee and the handling editor for their helpful comments and suggestions. W-T Li acknowledges support by the NNSF of China (10571078) and the Teaching and Research Award Program for Outstanding Young Teachers in Higher 
Education Institutions of Ministry of Education of China. S Ruan's research was partially supported by NSF grant DMS-0412047 and the University of Miami.

\section{References}

[1] Ashwin P B, Bartuccelli M V, Bridges T J and Gourly S A 2002 Travelling fronts for the KPP equation with spatio-temporal delay Z. Angew. Math. Phys. 53 103-22

[2] Al-Omari J and Gourly S A 2002 Monotone traveling fronts in age-structured reaction-diffusion model of a single species J. Math. Biol. 45 294-312

[3] Billingham J 2004 Dynamics of a strongly nonlocal reaction-diffusion population model Nonlinearity 17313-46

[4] Cantrell R S and Cosner G C 2003 Spatial Ecology via Reaction-Diffusion Equations (Chichester, UK: Wiley)

[5] Conley C and Gardner R 1984 An application of the generalized Mores index to traveling wave solutions of a competition reaction-diffusion model Indiana Univ. Math. J. 44 319-43

[6] Durrett R and Levin S 1994 The importance of being discrete (and spatial) Theor. Pop. Biol. 46 363-94

[7] Durrett R and Levin S 1998 Spatial aspects of interspecific competition Theor. Pop. Biol. 53 30-43

[8] Gardner R 1982 Existence and stability of traveling wave solutions of competition models: a degree theoretic approach J. Diff. Eqns $\mathbf{4 4} 343-64$

[9] Gourley S A and Ruan S 2003 Convergence and traveling fronts in functional differential equations with nonlocal terms: a competition model SIAM J. Math. Anal. 35 806-22

[10] Hanski I A and Gilpin M E 1996 Metapopulation Biology: Ecology, Genetics, and Evolution (New York: Academic)

[11] Hosono Y 1995 Traveling waves for a diffusive Lotka-Volterra competition model, II: a geometric approach Forma 10 235-57

[12] Hosono Y 2003 Traveling waves for a diffusive Lotka-Volterra competition model, I: singular perturbation Discrete Cont. Dyn. Syst. B 3 75-95

[13] Huang J, Lu G and Ruan S 2005 Traveling wave solutions in delayed lattice differential equations with partial monotonicity Nonlinear Anal. TMA 60 1331-50

[14] Huang J and Zou X 2002 Traveling wavefronts in diffusive and cooperative Lotka-Volterra system with delays J. Math. Anal. Appl. 271 455-66

[15] Huang J and Zou X 2003 Existence of traveling wavefronts of delayed reaction-diffusion systems without monotonicity Discrete Cont. Dyn. Syst. 9 925-36

[16] Kanel J I and Zhou L 1996 Existence of wave front solutions and estimates of wave speed for a competitiondiffusion system Nonlinear Anal. TMA 27 579-87

[17] Kan-on Y 1995 Parameter dependence of propagation speed of traveling waves for competition-diffusion equations SIAM J. Math. Anal. 26 340-63

[18] Kowalik M, Lipowski A and Ferreira A L 2002 Oscillations and dynamics in a two-dimensional prey-predator system Phys. Rev. E 66066107

[19] Lan K and Wu J 2003 Traveling wavefronts of reaction-diffusion equations with and without delays Nonlinear Anal. RWA 4 173-88

[20] Leung A W 1989 Systems of Nonlinear Partial Differential Equations with Applications to Biology and Engineering (Dordrecht: Kluwer)

[21] Li W T, Ruan S and Wang Z C 2005 On the diffusive Nicholson's Blowflies equation with nonlocal delays J. Nonlinear Sci. submitted

[22] Ma S 2001 Traveling wavefronts for delayed reaction-diffusion systems via a fixed point theorem J. Diff. Eqns $171294-314$

[23] Pao C V 1992 Nonlinear Parabolic and Elliptic Equations (New York: Plenum)

[24] Provata A, Nicolis G and Baras F 1999 Oscillatory dynamics in low-dimensional supports: a lattice LotkaVolterra model J. Chem. Phys. 110 8361-8

[25] Rauch E M and Bar-Yam Y 2006 Long-range interactions and evolutionary stability in a predator-prey system Phys. Rev. E 73020903

[26] Ruan S and Zhao X Q 1999 Persistence and extinction in two species reaction-diffusion systems with delays $J$. Diff. Eqns 156 71-92

[27] Schaaf K W 1987 Asymptotic behavior and traveling wave solutions for parabolic functional differential equations Trans. Am. Math. Soc. 302 587-615

[28] Silvertown J, Holtier S, Johnson J and Dale P 1992 Cellular automation models of interspecific competition for space-the effect of pattern on process J. Ecol. 80 527-34 
[29] Tang M M and Fife P 1980 Propagating fronts for competing species equations with diffusion Arch. Ration. Mech. Anal. 73 69-77

[30] van Vuuren J H 1995 The existence of traveling plane waves in a general class of competition-diffusion systems IMA J. Appl. Math. $\mathbf{5 5} 135-48$

[31] Wang Z C, Li W T and Ruan S 2006 Traveling wave fronts in reaction-diffusion systems with spatio-temporal delays J. Diff. Eqns 222 185-232

[32] Wu J 1996 Theory and Applications of Partial Functional Differential Equations (New York: Springer)

[33] Wu J and Zou X 2001 Traveling wave fronts of reaction-diffusion systems with delay J. Dyn. Diff. Eqns 13 651-87

[34] Ye Q X and Li Z Y 1994 Introduction to Reaction Diffusion Equations (Beijing: Science Press)

[35] Zeilder E 1986 Nonlinear Functional Analysis and its Applications: I, Fixed-Point Theorems (New York: Springer)

[36] Zou X and Wu J 1997 Existence of traveling wave fronts in delayed reaction-diffusion systems via the monotone iteration method Proc. Am. Math. Soc. 125 2589-98 